\title{
A visually secure image encryption scheme based on compressive sensing
}

\author{
Xiuli Chai ${ }^{1,2}$, Zhihua Gan ${ }^{3}$, Yiran Chen ${ }^{2}$, Yushu Zhang ${ }^{4}$ \\ 1 School of Computer and Information Engineering, Institute of Image Processing and Pattern \\ Recognition, Henan University, Kaifeng 475004, China \\ 2 Department of Electrical and Computer Engineering, University of Pittsburgh, Pittsburgh, PA
} 15261, United States

3 School of Software, Henan University, Kaifeng 475004, China

4 School of Electronics and Information Engineering, Southwest University, Chongqing 400715, China

Corresponding E-mail address: chaixiuli@henu.edu.cn (X. L. Chai)

\begin{abstract}
A novel visually secure image encryption scheme based on compressive sensing (CS) is proposed. Firstly, the plain image is transformed into wavelet coefficients, and then confused by a zigzag path and encrypted into a compressed cipher image using compressive sensing. Next, the cipher image is embedded into a carrier image, and finally a visually secure cipher image is obtained. SHA 256 hash function of the original image is generated to calculate the parameters for zigzag confusion and one-dimensional skew tent map, and the map is used to produce the measurement matrix for CS. Therefore, the proposed algorithm is highly sensitive to the plain image, and it can effectively withstand known-plaintext and chosen-plaintext attacks. Besides, our algorithm can achieve the image data security and image appearance security simultaneously, and the size of the cipher image and original image is equal, it does not require additional transmission
\end{abstract}


bandwidth and storage space. Simulation results and performance analyses both demonstrate excellent encryption performance of the proposed encryption scheme.

Keywords: Image encryption; compressive sensing (CS); image appearance security; skew tent map

\section{Introduction}

With the advancement of digital technologies and Internet, more and more digital images are generated, transmitted over the networks and stored on various platforms, such as cloud server, hard-drive, and others. Digital data may contain a large amount of information. For example, a military oil depot image can not only reflect its size and number, but also its location; a human face picture can not only tell his or her appearance, but also the rough age. Thus, protecting image has receiving increasingly interests in many fields, including medical imaging systems, military imaging systems, video conferencing. Image encryption is a very efficient scheme to keep data security.

Many image encryption algorithms have been introduced using chaotic system [1-5], DNA computing [6-8], cellular automata (CA) [9-11], optical transform [12-14], Brownian motion [15, 16], wave transmission [17, 18], Latin squares [19] and others. The common feature of the most existing image encryption schemes is that the plain image is transformed to a noise-like or texture-like cipher image by them [1-19], the histogram of the cipher image is uniform and flat, the information entropy is near 8 , the cipher image is transmitted from the sender to the receiver, and the hackers cannot obtain the original image and secret key through analyzing the cryptosystem. Most of the encryption methods can protect image data effectively, though some of 
them have been broken for that there is a small key space or the encryption schemes have little relationship with the original image [20-31]. Moreover, there is a common shortcoming for these encryption schemes, which is that noise-like or texture-like cipher images can easily catch hackers' attention during the transmission and storage, and then be attacked [32]. If we make the cipher image visually secure, the cipher image has a meaningful appearance, then when it is transmitted and stored with other images, the hackers cannot find it from many meaningful images, so that the cipher image or the corresponding plain image is more secure. Therefore, designing image data security and appearance security encryption algorithm is an important work. Meantime, we do not want the size of the cipher image is larger than that of the plain image, the increasing of the image size means more transmission bandwidth, more transmission time, and more memory space.

Recently, compressive sensing (CS) is applied in cryptography [33], it can unify sample, compress and encrypt the image simultaneously, and the measurement matrix can be considered as an encryption key. Some image compression and encryption algorithms using CS have been proposed [34-42]. For example, Fang et al [34] used zigzag-scan-based permutation and compressive sensing to compress and encrypt the image and video, and found that zigzag-based permutation increased the peak signal-to-noise ratio (PSNR) of reconstructed images and video frames. Zhang et al [35] gave an image compression and encryption scheme based on CS and the chaotic system, first, the sparse coefficients of the plain image were confused by chaotic sequence sorting, and then encrypted and compressed by CS, finally a noise-like cipher image was obtained. A novel image compression-encryption scheme was introduced by combining 2D compressive sensing and nonlinear fractional Mellin transform [36], the original image was measured by measurement matrices in two directions, and then re-encrypted by the nonlinear fractional Mellin 
transform. For the color image, Tong et al [37] proposed a chaos-based algorithm for joint image compression and encryption, first discrete cosine transformation dictionary was used to sparsely represent $R, G, B$ components of the color plain image, and then the subsequent confusion and diffusion processes based on the hyperchaotic system were employed. Xiao et al [38] introduced a robust watermarking of encrypted images based on compressive sensing and Arnold map, the image was scrambled with Arnold map and encrypted with CS, and then the watermark was embedded into the encrypted image. In these encryption algorithms, the combination of permutation process and compressive sensing can effectively relax the Restricted Isometry Constant for CS, reduce the storage and computation complexity in the decryption process, and improve the compression and encryption performance. But in the above encryption scheme, the permutation process has no relationship with the plain image, the same permutation vector is used for different original image, and the encryption algorithm may be easily broken by known-plaintext and chosen-plaintext attacks. So, increasing the dependence between the encryption scheme and the plain image is very necessary to upgrade the security level of the encryption algorithm.

Based on the above analyses, a novel visually secure image encryption algorithm using compressive sensing is presented. Our contributions are as follows. Firstly, image data security and image appearance security can be realized at the same time. The wavelet coefficients of the plain image are confused by a zigzag path, the confusion process is controlled by the plain image, and then the confused image is encrypted into a compressed cipher image based on compressive sensing, a plain image related measurement matrix for CS is generated using the one-dimensional skew tent map; and next the cipher image is embedded into a carrier image, the embedding 
process depends on the carrier image and finally a visually secure cipher image is gotten. Secondly, the cipher image can be transmitted and stored fast and efficiently. Combined CS and zigzag confusion, the original image is compressed and encrypted, the adoption of random zigzag confusion scheme can effectively relax the Restricted Isometry Constant for CS, and enhance the compression performance, and finally the cipher image has the same size with the original image, therefore, there is no extra transmission bandwidth and storage space. Besides, the SHA 256 hash function of the original image is used to compute the parameters for encryption, the key space is large, and the algorithm has a close relationship with the plain image, and it may resist against the known-plaintext and chosen-plaintext attacks.

The rest of the paper is organized as follows. In Section 2, some related works are given. In Section 3, the proposed encryption scheme is described. Numerical simulations are presented in Section 4. Performance analyses of the proposed algorithm are demonstrated in Section 5. And the last section concludes our work.

\section{Fundamental knowledge and related works}

\subsection{Compressive sensing}

In 2006, Candes and Donoho proposed the concept of compressive sensing (CS) [43, 44], it compresses and samples simultaneously, and allows the exact recovery of a sparse signal from some projections lower than the Nyquist rate.

Consider $\boldsymbol{x}$ to be a signal of dimensions $N \times 1, \boldsymbol{x} \in R^{n}$. To obtain $M$ nonadaptive linear measurements from $x$, we multiply it by a matrix $\boldsymbol{\Phi}$, and the sampling process is denoted as

$$
\boldsymbol{y}=\boldsymbol{\Phi} \boldsymbol{x}
$$

Where, $\boldsymbol{\Phi}$ is a measurement matrix with dimensions $M \times N, \boldsymbol{y}$ is a compressed 
measurement vector of dimension $M \times 1$, and $M \square N$

At most times, $\boldsymbol{x}$ is sparse in some sense or a certain transform domain (e.g. DCT, DWT), and it can be represented in transform domain $(\boldsymbol{\psi})$ as,

$$
\boldsymbol{x}=\sum_{i=1}^{N} \psi_{i} S_{i}=\boldsymbol{\psi s}
$$

Where $\psi_{i}$ is the $i$ th column vector of the $N \times N$ orthogonal basis matrix $\psi$, $\boldsymbol{s}=\left\{s_{1}, s_{2}, \cdots, s_{N}\right\}$ denotes $N \times 1$ coefficient vector.

Based on Eqs. (1) and (2), the overall sampling process is as follows,

$$
y=\Phi x=\Phi \psi s=A s
$$

Here, $\boldsymbol{A}$ is the $M \times N$ sensing matrix, and it is the product of $\boldsymbol{\Phi}$ and $\boldsymbol{\psi}$.

If $s$ is $K$-sparse, that means it has $K$ non-zero entries, only $K$ measurements are required for the exact reconstruction of $\boldsymbol{x}$. In order to reconstruct the signal $\boldsymbol{x}$ from $\boldsymbol{y}$ correctly, the matrix $\boldsymbol{A}$ should satisfy restricted isometry property (RIP) [43]. To meet the RIP condition, the measurement matrix can be selected as Gaussian random matrix, uniform random projection or partial random circular matrix. The problem of estimating the sparse signal $x$ can be computed by,

$$
\min \|\boldsymbol{s}\|_{0} \text { s.t. } \quad \boldsymbol{y}=\boldsymbol{\Phi} \psi \boldsymbol{s}
$$

Where, $\|\boldsymbol{s}\|_{0}$ denotes the $l_{0}$-norm of vector $\boldsymbol{s}$. The solution of the above problem is a NP-hard problem. To overcome it, the easy way is to convert it to a convex optimization problem, and Eq.(4) can be transformed to,

$$
\min \|\boldsymbol{s}\|_{1} \text { s.t. } \quad \boldsymbol{y}=\boldsymbol{\Phi} \psi \boldsymbol{s}
$$

Where, $\|\boldsymbol{s}\|_{1}$ denotes the $l_{1}$-norm of vector $\boldsymbol{s}$. Some reconstruction algorithms, such as matching pursuit (MP), orthogonal matching pursuit (OMP), smoothed $l_{0}$ norm $\left(\mathrm{SL}_{0}\right)$ are effective to recover $\boldsymbol{x}$ from $\boldsymbol{y}$. 


\subsection{Chaos-based measurement matrix}

For a random measurement matrix (MM), its storage and transmission need much space. Therefore, it is preferable to produce the measurement matrix using one or more seed keys. Yu et al [45] used the chaotic sequence from logistic map to construct the MM and proved that its RIP was guaranteed with overwhelming probability. In the paper, the one-dimensional skew tent map [46] is used to generate MM, its probability density function is more uniform compared with logistic map, and it is given by

$$
z(k+1)=T[z(k) ; r]=\left\{\begin{array}{l}
\frac{z(k)}{r}, 0<z(k)<r \\
\frac{1-z(k)}{1-r}, r \leq z(k)<1
\end{array}\right.
$$

Here, the system parameter $r \in(0,1)$ and the initial value $z_{0} \in(0,1)$.

Let $z\left(d, l, r, z_{0}\right)=(z(n+i \times d))_{i=0}^{l}=\left(z_{n}, z_{n+d}, z_{n+2 d}, \cdots, z_{n+l d}\right)$ be the chaotic sequence sampled from the output sequence produced by Eq. (6), $d$ is the sampling distance, and $r, z_{0}$ are the corresponding parameter and initial value of the skew tent map.

Next, perform the following transformation on the chaotic sequence as Eq. (7),

$$
\left.\varphi(l)\right|_{l=0} ^{M N-1}=\left.\left(1-2 z_{n+l d}\right)\right|_{l=0} ^{M N-1}
$$

To construct the measurement matrix $\boldsymbol{\Phi} \in R^{M \times N}$, the sampled chaotic sequence $z\left(d, l, r, z_{0}\right)$ with the length $l=M N$ is generated, and using this sequence $\left.\varphi(l)\right|_{l=0} ^{M N-1}$, a matrix

$\boldsymbol{\Phi}$ is created and illustrated as

$$
\boldsymbol{\Phi}=\sqrt{\frac{2}{M}}\left[\begin{array}{ccc}
\varphi(0) & \cdots & \varphi(M(N-1)) \\
\varphi(1) & \cdots & \varphi(M(N-1)+1) \\
\cdots & \cdots & \cdots \\
\varphi(M-1) & \cdots & \varphi(M N-1)
\end{array}\right]
$$

Where, $\sqrt{\frac{2}{M}}$ is used for normalization. 
From the generation process of chaotic measurement matrix, we can conclude that firstly, the measurement matrix for compressive sensing can be produced using some key parameters, the sender transmits these key parameters to the receiver, and the receiver can get the same measurement matrix for recovering the original image, but in the past, the sender must transmit the measurement matrix to the receiver for decryption, compared with it, our proposed algorithm can save much transmission bandwidth and storage space; secondly, through modifying the system parameter $r$ and initial value $z_{0}$ of the chaotic system, different measurement matrices can be generated; lastly, chaotic systems have the feature of sensitivity to the parameter and initial values, good randomness, we adopt the one-dimensional skew tent map to generate the measurement matrix, which can improve the security level of the encryption algorithm.

\subsection{Zigzag confusion}

A zigzag path [47] shown in Fig.1 is used to confuse the sparse coefficient matrix of the plain image, and it can disturb the high correlation among image pixels to increase the security level of the encryption algorithm. For zigzag confusion, location of the starting pixel in the matrix is very important, different location can generate different confusion effect. For example, if the location of the starting pixel is $(1,1)$, that means we start traversing the path from the first pixel, and the matrices before and after zigzag confusion are shown in Fig.2. If the location is $(3,4)$, which means that we start traversing the path of the matrix from pixel number $3 \times 4=12$, and the matrices before and after zigzag confusion are illustrated in Fig.3, and in Fig.3, the 12th pixel is 160. In the paper, in order to improve the dependence of the encryption algorithm on the original image, we calculate the starting location of zigzag confusion according to the plain image, and then different plain image has different confusion effect, which can upgrade the capability of resisting 
known-plaintext and chosen-plaintext attacks.

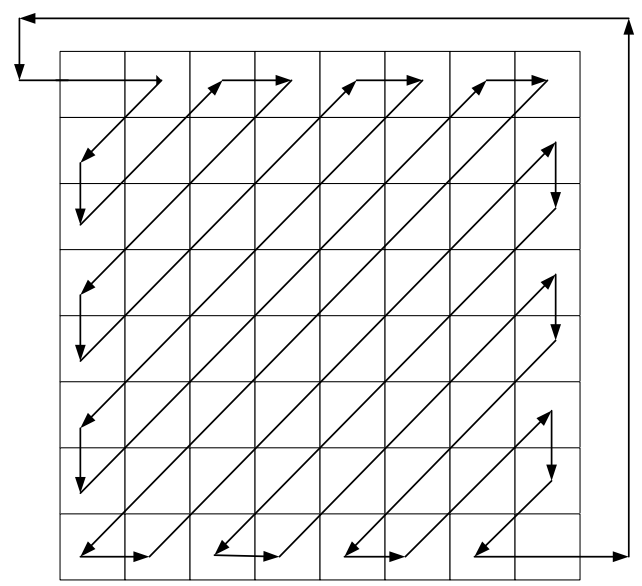

Fig.1 A zigzag path for confusion

\begin{tabular}{|c|c|c|c|c|c|c|c|c|c|c|c|c|c|c|c|}
\hline 1 & 2 & 6 & 7 & 15 & 16 & 28 & 29 & 1 & 2 & 3 & 4 & 5 & 6 & 7 & 8 \\
\hline 3 & 5 & 8 & 14 & 17 & 27 & 30 & 43 & 9 & 10 & 11 & 12 & 13 & 14 & 15 & 16 \\
\hline 4 & 9 & 13 & 18 & 26 & 31 & 42 & 44 & 17 & 18 & 19 & 20 & 21 & 22 & 23 & 24 \\
\hline 10 & 12 & 19 & 25 & 32 & 41 & 45 & 54 & 25 & 26 & 27 & 28 & 29 & 30 & 31 & 32 \\
\hline 11 & 20 & 24 & 33 & 40 & 46 & 53 & 55 & 33 & 34 & 35 & 36 & 37 & 38 & 39 & 40 \\
\hline 21 & 23 & 34 & 39 & 47 & 52 & 56 & 61 & 41 & 42 & 43 & 44 & 45 & 46 & 47 & 48 \\
\hline 22 & 35 & 38 & 48 & 51 & 57 & 60 & 62 & 49 & 50 & 51 & 52 & 53 & 54 & 55 & 56 \\
\hline 36 & 37 & 49 & 50 & 58 & 59 & 63 & 64 & 57 & 58 & 59 & 60 & 61 & 62 & 63 & 64 \\
\hline
\end{tabular}

Fig.2 The matrices before and after zigzag confusion with the starting pixel $(1,1)$

\begin{tabular}{|l|l|l|l|l|l|l|l|}
\hline 210 & 239 & 195 & 197 & 240 & 68 & 2 & 151 \\
\hline 142 & 168 & 243 & 42 & 100 & 205 & 3 & 203 \\
\hline 60 & 142 & 154 & 240 & 187 & 150 & 45 & 122 \\
\hline 206 & 160 & 20 & 49 & 46 & 68 & 55 & 144 \\
\hline 74 & 237 & 227 & 28 & 18 & 89 & 255 & 8 \\
\hline 150 & 103 & 218 & 250 & 136 & 153 & 220 & 90 \\
\hline 216 & 34 & 123 & 162 & 171 & 204 & 87 & 216 \\
\hline 245 & 76 & 115 & 108 & 63 & 5 & 110 & 14 \\
\hline
\end{tabular}$\quad$\begin{tabular}{|l|l|l|l|l|l|l|l|l|}
\hline 160 & 154 & 42 & 240 & 68 & 100 & 240 & 20 \\
\hline 237 & 150 & 216 & 103 & 227 & 49 & 187 & 205 \\
\hline 2 & 151 & 3 & 150 & 46 & 28 & 218 & 34 \\
\hline 245 & 76 & 123 & 250 & 18 & 68 & 45 & 203 \\
\hline 122 & 55 & 89 & 136 & 162 & 115 & 108 & 171 \\
\hline 153 & 255 & 144 & 8 & 220 & 204 & 63 & 5 \\
\hline 87 & 90 & 216 & 110 & 14 & 210 & 239 & 142 \\
\hline 60 & 168 & 195 & 197 & 243 & 142 & 206 & 74 \\
\hline
\end{tabular}

Fig.3 The matrices before and after zigzag confusion with the starting pixel $(3,4)$

\section{The proposed encryption scheme}

\subsection{The generation of parameters}

In order to increase the relationship between the encryption scheme and the plain image, we employ the related information of the original image to determine the parameters. The important parameters consist of the location $\left(x_{0}, y_{0}\right)$ of the starting pixel for zigzag confusion, and the 
parameters $r, z_{0}$ of the skew tent map. Firstly, we obtain 256-bit secret key $K$ through the SHA 256 hash function of the plain image, then it is divided into 8-bit blocks, so it can be expressed as follows:

$$
K=k_{1}, k_{2}, \cdots, k_{32} \text {, subject to: } k_{i}=\left\{k_{i, 0}, k_{i, 1}, \cdots, k_{i, 7}\right\}
$$

Where in $k_{i, j}, i$ denotes the character number and $j$ is the bit number in $k_{i}$.

Next, the four intermediate parameters $t_{\mathrm{i}}$ are computed by,

$$
t_{i}=\left[l_{i}+\left(\frac{k_{4 i-2}+(4 i-2)}{k_{3 i}+3 i}+\frac{k_{5 i-3}+(5 i-3)}{k_{4 i}+4 i}\right) \times \sum_{j=1}^{32} \frac{k_{j} \times 2^{8 \times(j-1)}}{2^{256}}\right] \bmod 1, i=1,2,3,4
$$

Here, $l_{i}(i=1,2,3,4)$ are the given parameters for individually denoting the parameters $x_{0}^{\prime}$, $y_{0}^{\prime}, r^{\prime}, z_{0}^{\prime}, t_{3}$ and $t_{4}$ individually denote the parameters $r, z_{0}$, and $x_{0}, y_{0}$ can be given by

$$
\left\{\begin{array}{l}
x_{0}=\left\lfloor\left(\operatorname{abs}\left(t_{1}\right)-\left\lfloor t_{1}\right\rfloor\right) \times 10^{14}\right\rfloor \bmod m+1 \\
y_{0}=\left\lfloor\left(a b s\left(t_{2}\right)-\left\lfloor t_{2}\right\rfloor\right) \times 10^{14}\right\rfloor \bmod n+1
\end{array}\right.
$$

Where, $a b s(x)$ is the absolute value of $x$, and mod is the modular operator.

The parameters $x_{0}^{\prime}, y_{0}^{\prime}, r^{\prime}, z_{0}^{\prime}$ are changeable, and given as secret keys, so that even for the same plain image, the 256-bit hash value is fixed, but we can obtain different parameters $r, z_{0}, x_{0}$, $y_{0}$ by modifying $x_{0}^{\prime}, y_{0}^{\prime}, r^{\prime}, z_{0}^{\prime}$, and “one time, one key” can be realized.

\subsection{The encryption algorithm}

In this section, a new visually secure image encryption method based on compressive sensing is proposed. The proposed encryption scheme is composed of two stages. In the first stage, the plain image is compressed and encrypted by zigzag confusion and compressive sensing. Then, at the second stage, the cipher image is embedded into the carrier image, and a visually secure cipher image is obtained. As shown in Fig. 4, the encryption steps can be described as follows. 


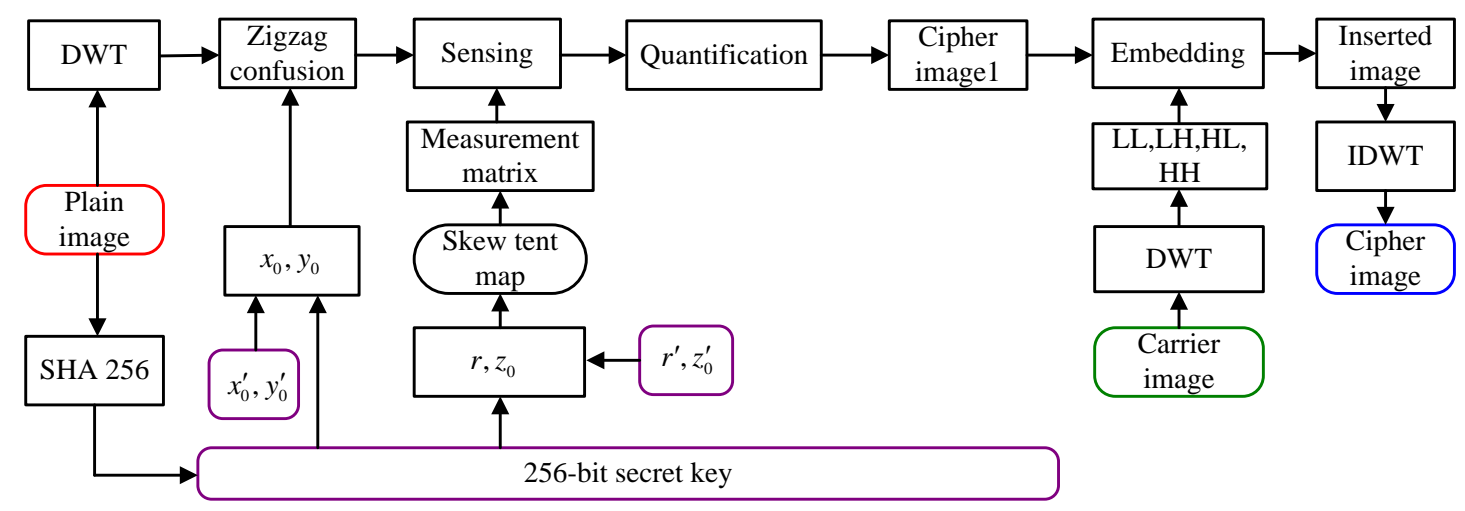

Fig.4 The flow chart of the proposed encryption algorithm

\subsubsection{Compressing and encrypting the plain image based on zigzag confusion and CS}

The sender compresses and encrypts the plain image into a cipher image, and the detailed steps are as follows.

Step 1: Compute the SHA 256 hash function of the plain image, then 256-bit secret key $K$ is obtained and the four parameters $x_{0}, y_{0}, r, z_{0}$ are calculated with the given parameters $x_{0}^{\prime}, y_{0}^{\prime}, r^{\prime}, z_{0}^{\prime}$ as shown in Section 3.1.

Step 2: Assume the size of the plain image $P$ is $m \times n$, then it is sparsified using discrete wavelet transform (DWT), and the coefficient matrix $P 1$ with the same size is obtained.

Step 3: Perform zigzag confusion on $P 1$ with $x_{0}, y_{0}$ as described in Section 2.3, and the confused matrix $P 2$ is gotten. Next, set a threshold TS, modify the elements of $P 2$, and change them smaller than TS into 0 , and matrix $P 3$ is obtained.

Step 4: Generate the measurement matrix $\boldsymbol{\Phi}$ using $r, z_{0}$ according to Section 2.2, and then apply compressive sensing to matrix $P 3$, and obtain the measurement value matrix $P 4$. Set the compression ratio of the plain image is $C R$, then the size of $\boldsymbol{\Phi}$ is $M \times N$, and $M=C R \times m, N=m$. Correspondingly, the size of $P 4$ is $M \times n$.

Step 5: Quantifying the elements of matrix $P 4$, and convert the element values to the range of 0 
and 255 according to Eq. (12), and the matrix $P 5$ is obtained, and it is the compressed cipher image 1 of the plain image.

$$
p_{5 i}=\text { floor }\left(255 \times\left(p_{4 i}-\min \right) /(\max -\min )\right)
$$

Where, min is the minimum value of matrix $P 4$, max is the maximum value of $P 4$, floor $(x)$ calculates the largest integer that is no more than $x$, and $p_{4 \mathrm{i}}$ and $p_{5 \mathrm{i}}$ are the $i$ th elements of $P 4$ and $P 5(1 \leq i \leq M n)$, respectively.

\subsubsection{Embedding the cipher image into the carrier image}

Next, we embed the cipher image 1 (matrix $P 5$ ) into the carrier image, finally obtain the visually secure cipher image, and the detailed steps are illustrated as below.

Step 1: In order to safely embed the matrix $P 5$ into a carrier image, firstly, we transform its element values according to Eqs. (13) and (14), and two matrices $D 1$ and $D 2$ are gotten.

$$
\begin{aligned}
& D 1(i, j)=P 5(i, j) \bmod 10 \\
& D 2(i, j)=\text { floor }(P 5(i, j) / 10)
\end{aligned}
$$

Where, $D 1(i, j), D 2(i, j)$ and $P 5(i, j)$ are the elements of matrices $D 1, D 2$ and $P 5$ located at $(i, j), 1$ $\leq i \leq M, 1 \leq j \leq n$, respectively.

Step 2: Assume the size of the carrier image $C I$ is $m \times n$, in order to perfectly embed the cipher image $P 5$ into $C I$, we set the compression ratio $C R$ of the plain image as $C R=0.25$, then the size of the matrices $D 1, D 2$ and $P 5$ are all $(m / 4) \times n$, and then $D 1, D 2$ are resized to matrices with the size of $(m / 2) \times(n / 2)$.

Step 3: Apply DWT to the carrier image $C I$, four matrices $L L, L H, H L$ and $H H$ are obtained, and their sizes are all $(m / 2) \times(n / 2)$.

Step 4: Define an empty matrix IT with the size of $(m / 2) \times(n / 2)$, and then calculate the 
medium value $M V$ of all elements of $L L$ matrix, and next embed $D 1, D 2$ into the $L H$ and $H L$ as follows:

Case 1: If $L L(i, j) \geq M V$, then $I T(i, j)=1, L H(i, j)=D 1(i, j), H L(i, j)=D 2(i, j)$.

Case 2: If $I T(i, j)<M V$, then $I T(i, j)=0, L H(i, j)=D 2(i, j), H L(i, j)=D 1(i, j)$.

Step 5: Manipulate inverse discrete wavelet transform (IDWT) to the combination of $L L, L H, H L$ and $H H$, and a visually secure cipher image $C$ is obtained. The encryption process is finished.

\subsection{The decryption algorithm}

The decryption algorithm is the inverse operation of the encryption, and the flow chart is shown in Fig. 5. It is composed of two stages, and they are extracting the cipher image 1 of the plain image from the visually secure cipher image, and then recovering the plain image. Before the decryption, secret keys including 256-bit hash value $K, x_{0}^{\prime}, y_{0}^{\prime}, r^{\prime}, z_{0}^{\prime}$, two parameters: $\min$ and max are transmitted to the receiver. And the four parameters $x_{0}, y_{0}, r, z_{0}$ are firstly computed as described in Section 3.1.

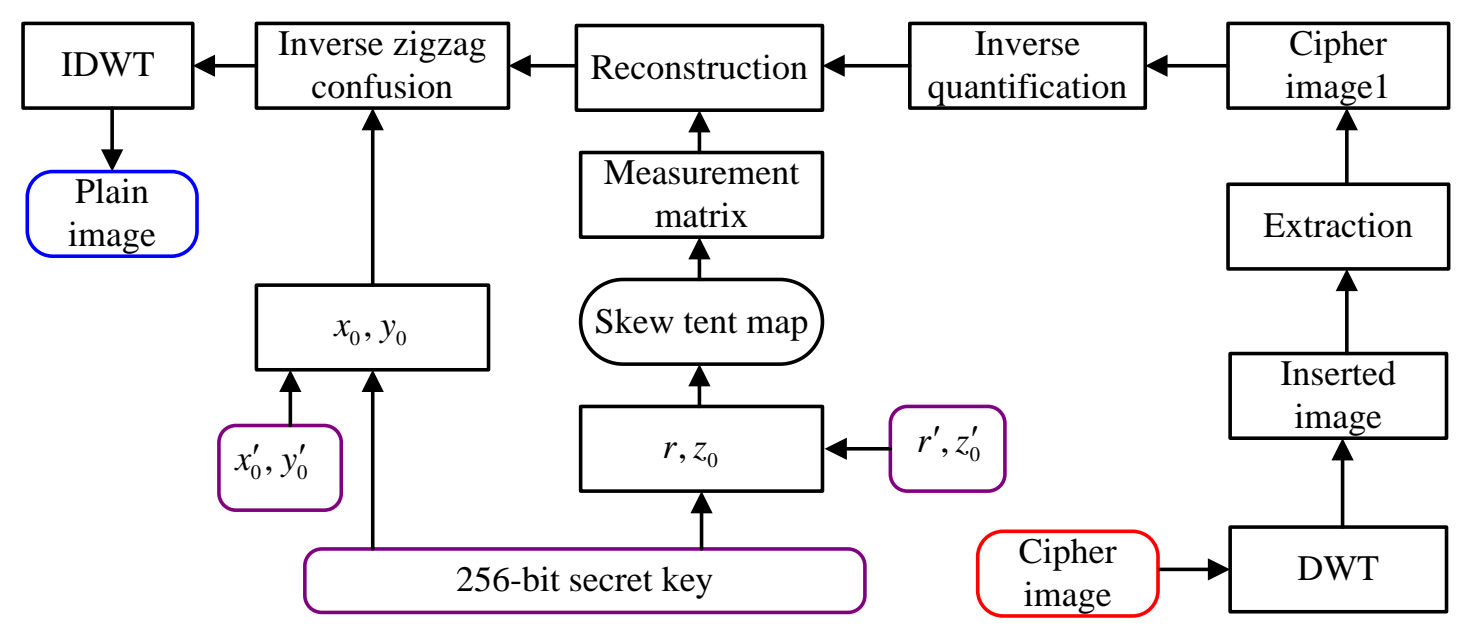

Fig.5 The flow chart of the proposed decryption algorithm

\subsubsection{Extracting the compressed cipher image from the visually secure cipher image}

Assume the visually secure cipher image is $C(m \times n)$, we first extract the compressed cipher 
image of the original image from it, and the detailed steps are as follows.

Step 1: Perform DWT to $C(m \times n)$, and get four matrices $L L, L H, H L$ and $H H$.

Step 2: Give an empty matrix IT with the size of $(m / 2) \times(n / 2)$, and then compute the medium value $M V$ of $L L$ matrix, and extract $D 1, D 2$ from the $L H$ and $H L$ as follows:

Case 1: If $L L(i, j) \geq M V$, then $I T(i, j)=1, D 1(i, j)=L H(i, j), D 2(i, j)=H L(i, j)$.

Case 2: If $I T(i, j)<M V$, then $I T(i, j)=0, D 2(i, j)=L H(i, j), D 1(i, j)=H L(i, j)$.

Step 3: Resize $D 1, D 2$ into matrices of $(m / 4) \times n$, the compressed cipher image $P 5$ of the plain image is obtained by the following formula,

$$
P 5(i, j)=D 2(i, j) \times 10+D 1(i, j)
$$

Here, $D 1(i, j), D 2(i, j)$ and $P 5(i, j)$ denote the elements of matrices $D 1, D 2$ and $P 5$ located at $(i, j)$, $1 \leq i \leq m / 4,1 \leq j \leq n$, respectively.

\subsubsection{Recovering the plain image}

Next, we reconstruct the plain image from the cipher image $P 5$, and the detailed steps are as below.

Step 1: Perform inverse quantification process on $P 5$ according to Eq. (16), and a matrix $P 4$ is obtained and it is the measurement value matrix.

$$
p_{4 i}=\frac{p_{5 i} \times(\max -\min )}{255}+\min
$$

Where, $p_{4 \mathrm{i}}$ and $p_{5 \mathrm{i}}$ are the $i$ th element of $P 4$ and $P 5(1 \leq i \leq M n)$, respectively, and min and max are gotten as secret key from the sender.

Step 2: Produce the measurement matrix $\boldsymbol{\Phi}$ using $r, z_{0}$ according to Section 2.2, and obtain matrix $P 3$ from $P 4$ using a certain reconstruction algorithm.

Step 3: Manipulate inverse zigzag confusion on $P 3$ with $x_{0}, y_{0}$, and the matrix $P 1$ is obtained. And 
finally, the original image $P$ is obtained by applying inverse discrete wavelet transform (IDWT) to $P 1$. And the decryption process is finished.

\subsection{Discussion}

The proposed image encryption scheme has some merits.

Firstly, a novel visually secure image encryption scheme is proposed. Using zigzag confusion and compressive sensing to compress and encrypt the plain image, a compressed cipher image is obtained, then we embed it into a carrier image, a meaningful cipher image is obtained, and transmitted across the internet and stored. Compared with the current noise-like or texture-like cipher images, the meaningful cipher image cannot be found easily by attackers and attain the image appearance security.

Secondly, the proposed encryption algorithm can make the plain image data secure. In the encryption process, the sparse coefficient matrix of the original image is confused by zigzag confusion, and the confusion process is controlled by the plain image. The confusion process can not only decrease the correlation between adjacent pixels, but also enhance reconstruction performance at the same compression ratio. Next, the confusion matrix is encrypted by compressive sensing, and keys generated by the plain image are used to produce the measurement matrix for CS. Moreover, in the embedding process, firstly, a carrier image is manipulated DWT operation, then the cipher image pixel values are divided into two parts, and they are embedded into $L H$ and $H L$ of the carrier image according to the element values of $L L$ pixel by pixel. All these schemes can ensure the high security level of the proposed encryption algorithm.

Thirdly, the proposed encryption scheme is highly sensitive to the plain image. The SHA 256 hash value of the plain image is used to calculate four important parameters $x_{0}, y_{0}, r, z_{0} . x_{0}, y_{0}$ are 
employed in the zigzag confusion process, and the confusion result is accordingly changed when parameters are changed with the original image. $r, z_{0}$ are utilized for designing the measurement matrix for CS, and different measurement matrix are obtained for different plain image. Therefore, our algorithm has not only large key space to resist the brute force attack, but also high sensitivity to the plain image and it can withstand known-plaintext and chosen-plaintext attacks.

Fourthly, the carrier image can be chosen in a variety of range. In view of the complete encryption process, the compression ratio $C R$ of the plain image is determined by the size of the plain image $P$ and the carrier image $C I$. In the above encryption scheme, the size of the original image is the same with that of the carrier image, and $C R=0.25$. If the size of the original image $P$ is $m \times n$, the size of $C I$ is $m 1 \times n 1$, then $C R=(m 1 \times n 1) / 4 m n$. That means the carrier image can be chosen freely, and its size can be changeable. When the size of the plain image is constant, the $C R$ is larger, that means more data are sampled from the plain image, and thus the construction quality of the plain image is higher. However, the larger carrier image needs more encryption and decryption time, more storage space and transmission bandwidth. Therefore, we should pick out the appropriate carrier image for all concerned.

Lastly, the decryption process is easy to manipulate. The plain image can be recovered without knowing the carrier image, which means the carrier image in the encryption process need not be transmitted to the receiver for decryption, and this can relieve the pressure of channel transmission and storage space. Besides, in our encryption scheme, the size of the plain image and final cipher image is equal, and there is no additional memory space. In future, when the compression performance of compressive sensing is improved, the size of the carrier image and final cipher image will become smaller. 


\section{Simulation results}

\subsection{Encryption results and decryption results for Lena $(512 \times 512)$ and Finger $(256 \times 256)$}

In this section, we have employed Matlab 2014a to verify the encryption and decryption effect of the proposed algorithm in a personal computer with 3GHz CPU and 4GB memory, the operating system is Microsoft Windows 7. The parameters we used are as follows: $x_{0}^{\prime}=0.2796$, $y_{0}^{\prime}=0.7531, r^{\prime}=0.5678, z_{0}^{\prime}=0.8652$, and the sampling distance $d$ is 20 , threshold $T S=50$. In the DWT decomposition of the plain image and carrier image, the "Haar" wavelet function is used. In the reconstruction process of the $\mathrm{CS}$, smoothed $l_{0}$ norm $\left(\mathrm{SL}_{0}\right)$ is employed.

Fig.6 is the simulation results of the plain image Lena $(512 \times 512)$, and the same size carrier image is Baboon shown in Fig.6(c). Fig.7 is the encryption and decryption results of the plain image Finger $(256 \times 256)$, and the carrier image is Cameraman $(256 \times 256)$. As are illustrated in Fig.6 and Fig.7 that Fig.6(b) and Fig.7(b) are the compressed cipher images of the plain images Lena and Finger, they are noisy, when they are transmitted over the Internet, they can be easily found and attacked by the hackers. Moreover, Fig.6(d) and Fig.7(d) are the corresponding visually secure cipher images, they are meaningful and may not be taken as cipher images from the appearance and have relative highly appearance security level. Besides, from visual point, the decrypted images shown in Fig.6(e) and Fig.7(e) are just the same as the respective plain images illustrated in Fig.6(a) and Fig.7(a). Therefore, the proposed encryption algorithm has good encryption and decryption effect, it can attain the image data security and appearance security, and the visually secure cipher image generated by the encryption method has the same size with the original image, there is no additional transmission bandwidth and memory. 


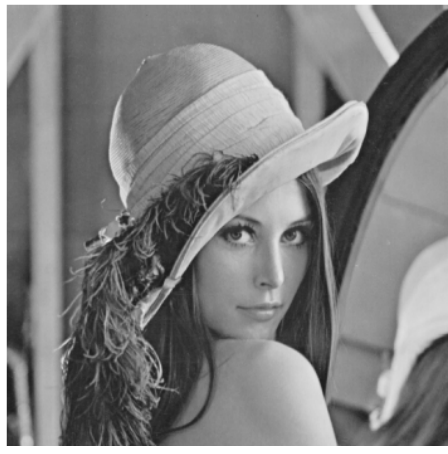

(a) Plain image Lena

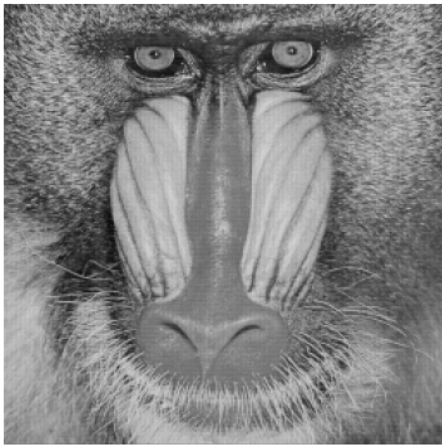

(d) Visually secure cipher image

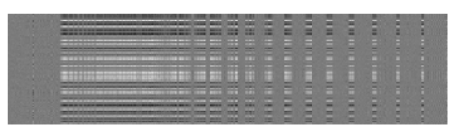

(b) Cipher image 1

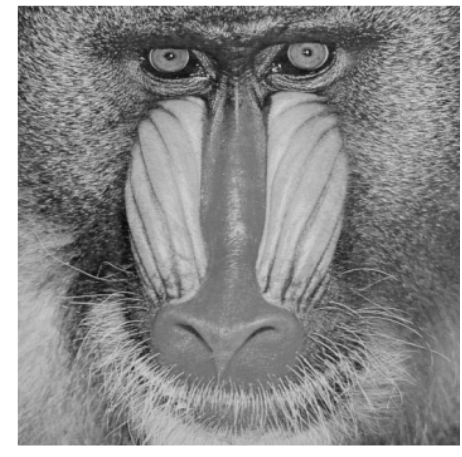

(c) Carrier image

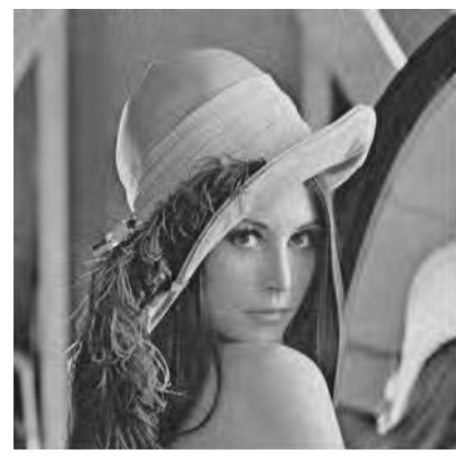

(e) Decrypted image

Fig. 6 Encryption and decryption results of the plain image Lena $(512 \times 512)$

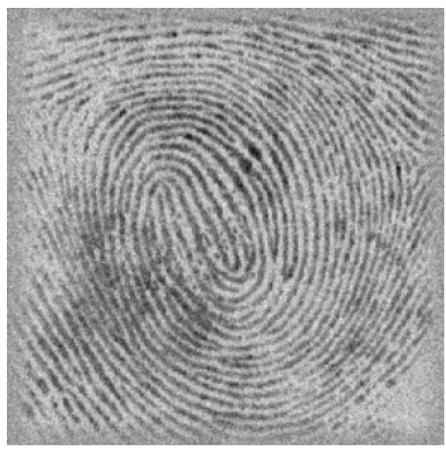

(a) Plain image Finger

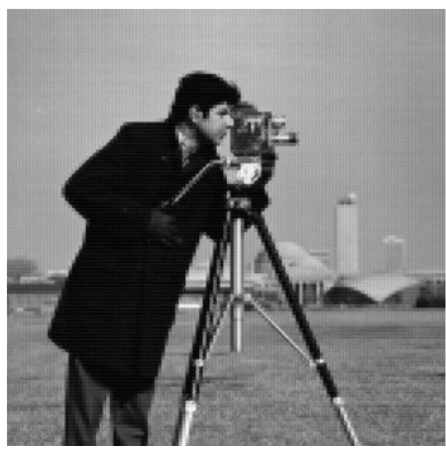

(d) Visually secure cipher image

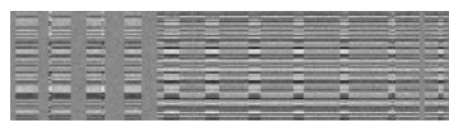

(b) Cipher image 1

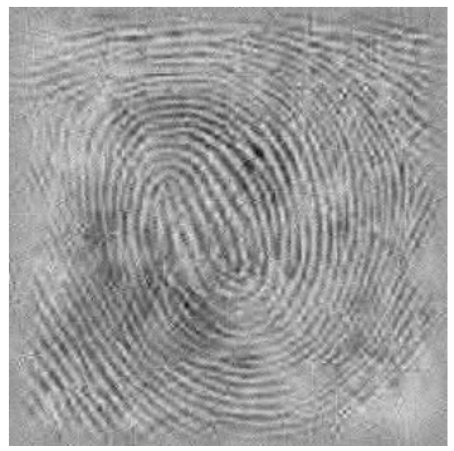

(e) Decrypted image

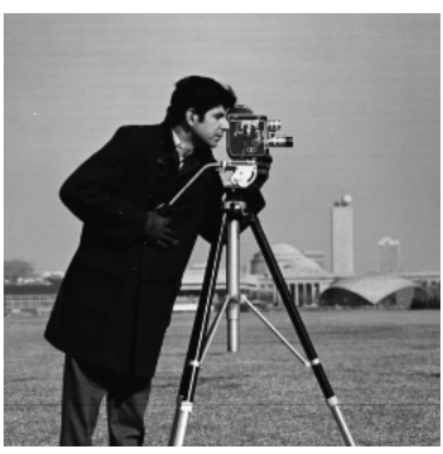

(c) Carrier image

Fig. 7 Encryption and decryption results of the plain image Finger $(256 \times 256)$ 


\subsection{The relationship between encryption and decryption results and three important}

parameters

The sparsifying methods of the plain image, the reconstruction methods of the CS, and threshold TS for sparse coefficient transformation of the plain image will affect the encryption and decryption results. Next, we will analyze the effect of them on the simulation results. The plain image is Peppers $(512 \times 512)$, the carrier image is Barbara $(512 \times 512)$, the sparsifying methods include DWT and DCT2, the reconstruction methods are $\mathrm{OMP}$ and $\mathrm{SL}_{0}$, and $T S$ is changing from 0 to 200. The encryption results are shown in Fig.8, and TS is 50. In Fig.8, (a) is the plain image Peppers, (b) is the carrier image Barbara, (c) is the visually secure cipher image 1 with DWT, (d) is the visually secure cipher image 2 with DCT2, and (e), (f), (g), (h) are their corresponding histograms. From Fig.8, it can be seen that (c) and (d) have no clear difference from the appearance, they are just like (b), and the appearance security of the cipher image can be attained with DWT or DCT 2.

Fig.9 gives the decryption results with different reconstruction methods. (a) is the decrypted image of Fig.8(c) with $\mathrm{SL}_{0}$, (b) is the decrypted image of Fig.8(c) with OMP, and (c) and (d) are the corresponding decrypted image of Fig.8 (d) with $\mathrm{SL}_{0}$, OMP. Fig.9 (e), (f), (g), (h) are their histograms, respectively. It is clear that when the plain image is using DWT, the recovered images both have high reconstruction quality whether using $\mathrm{SL}_{0}$ or $\mathrm{OMP}$, and they are just like the plain image; when the plain image employs DCT2 in the encryption process, the decrypted images are visually visible and have worse reconstruction quality. 


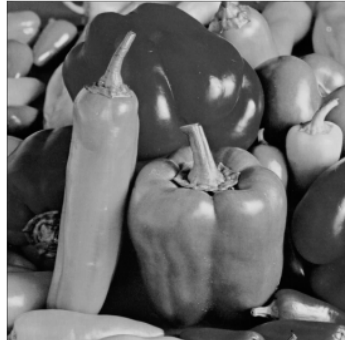

(a) Peppers $(512 \times 512)$

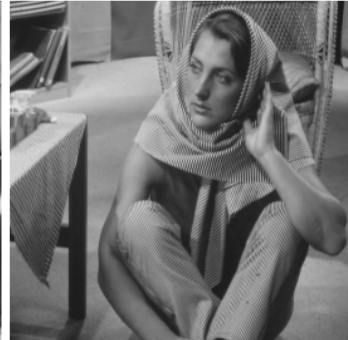

(b) Barbara

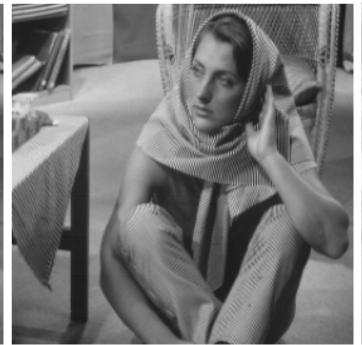

(c) Cipher image 1

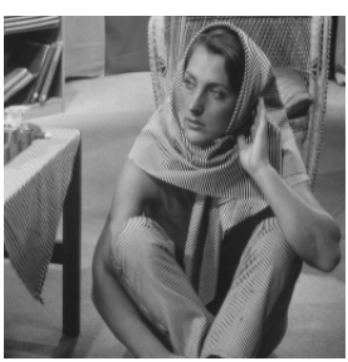

(d) Cipher image 2

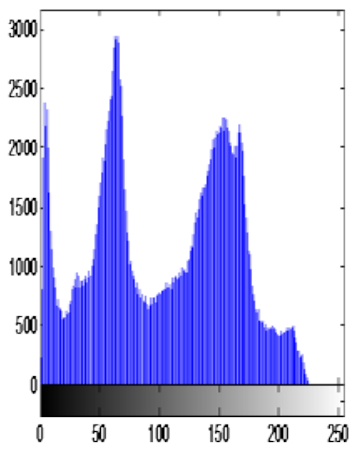

(e) Histogram of (a)

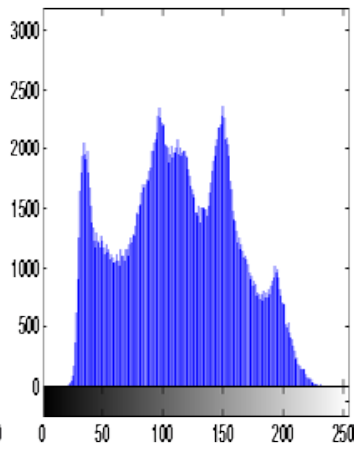

(f) Histogram of (b)

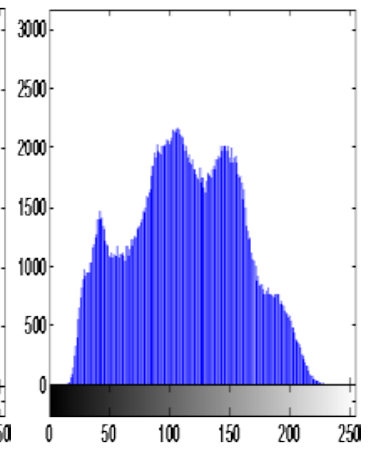

(g) Histogram of (c)

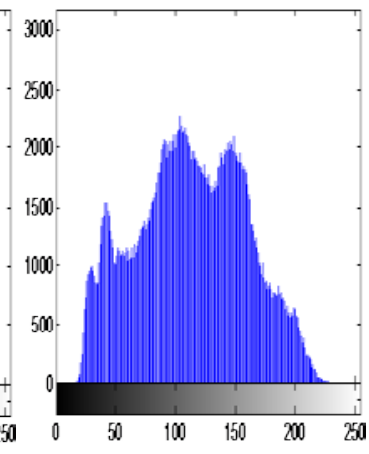

(h) Histogram of (d)

Fig.8 Encryption results with different sparsifying methods: (a) plain image Peppers, (b) carrier image Barbara, (c) visually secure cipher image 1 with DWT, (d) visually secure cipher image 2 with DCT2, (e) histogram of (a), (f) histogram of (b), (g) histogram of (c), (h) histogram of (d).

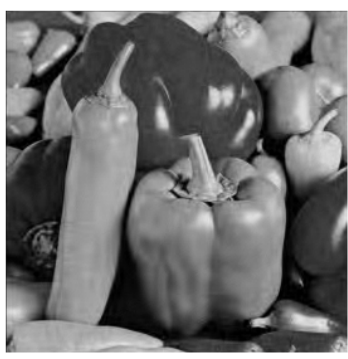

(a) Decrypted image

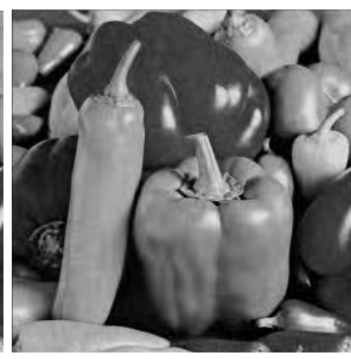

(b) Decrypted image

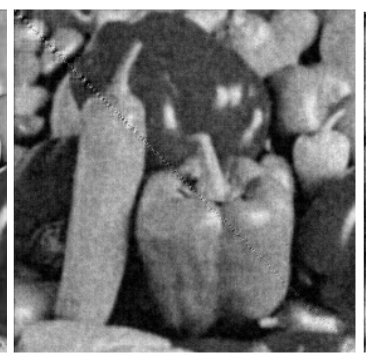

(c) Decrypted image

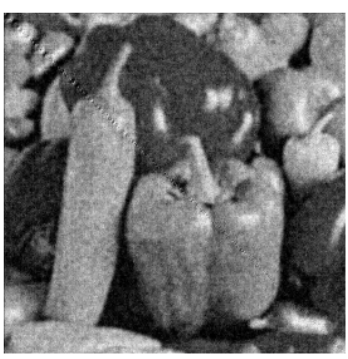

(d) Decrypted image

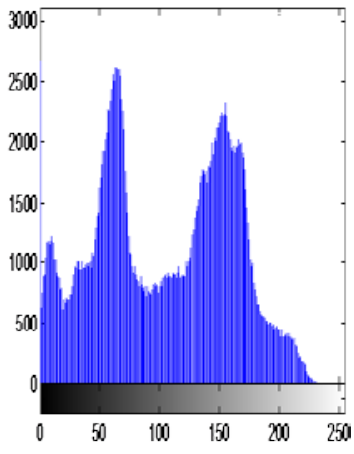

(e) Histogram of (a)

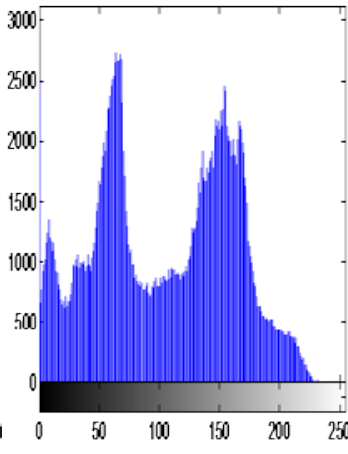

(f) Histogram of (b)

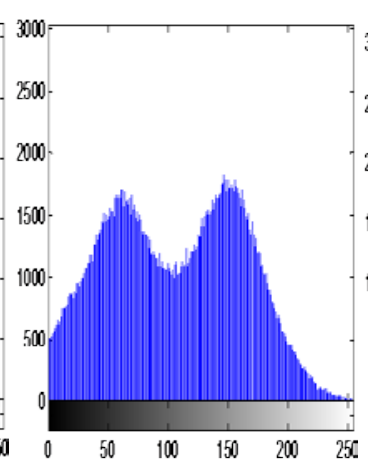

(g) Histogram of (c)

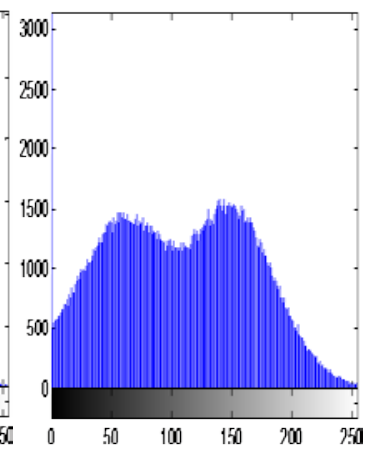

(h) Histogram of (d)

Fig.9 Decrypted results with different reconstruction methods: (a) decrypted image of Fig.8(c) 
with $\mathrm{SL}_{0}$, (b) decrypted image of Fig.8(c) with OMP, (c) decrypted image of Fig.8 (d) with $\mathrm{SL}_{0}$, (d) decrypted image of Fig.8 (d) with OMP, (e) histogram of (a), (f) histogram of (b), (g) histogram of (c), (h) histogram of (d).

Fig.10 plots the relationship of PSNR and TS for Peppers with different sparsifying and reconstruction methods. From the figure, we can see that (1) when $T S=-1$, and there is without sparse coefficient transformation, the PSNR values of the decrypted image and plain image are the same, which means that after the original image is sparsified with DWT or DCT2, and the coefficients are very sparse with many values near 0 ; (2) when the original image is sparsified with DCT2, the PSNR values are below $30 \mathrm{~dB}$, when the TS is varying from 0 to 200 , the changing of TS has little effect on PSNR values, and using $\mathrm{SL}_{0}$ has better decryption effect than using OMP; (3) when the plain image employs DWT, we can get the largest PSNR values when TS is around 20 , and it is $36.30 \mathrm{~dB}$ with OMP and $35.37 \mathrm{~dB}$ with $\mathrm{SL}_{0}$, and then with the decreasing of TS, PNSR values drop sharply, and they are $31 \mathrm{~dB}$ with $T S=200$. All in all, in order to get better decrypted images, the sparsifying method should adopt DWT, and the reconstruction method can use OMP or $\mathrm{SL}_{0}$, and we can pick out a value around 20 as TS.

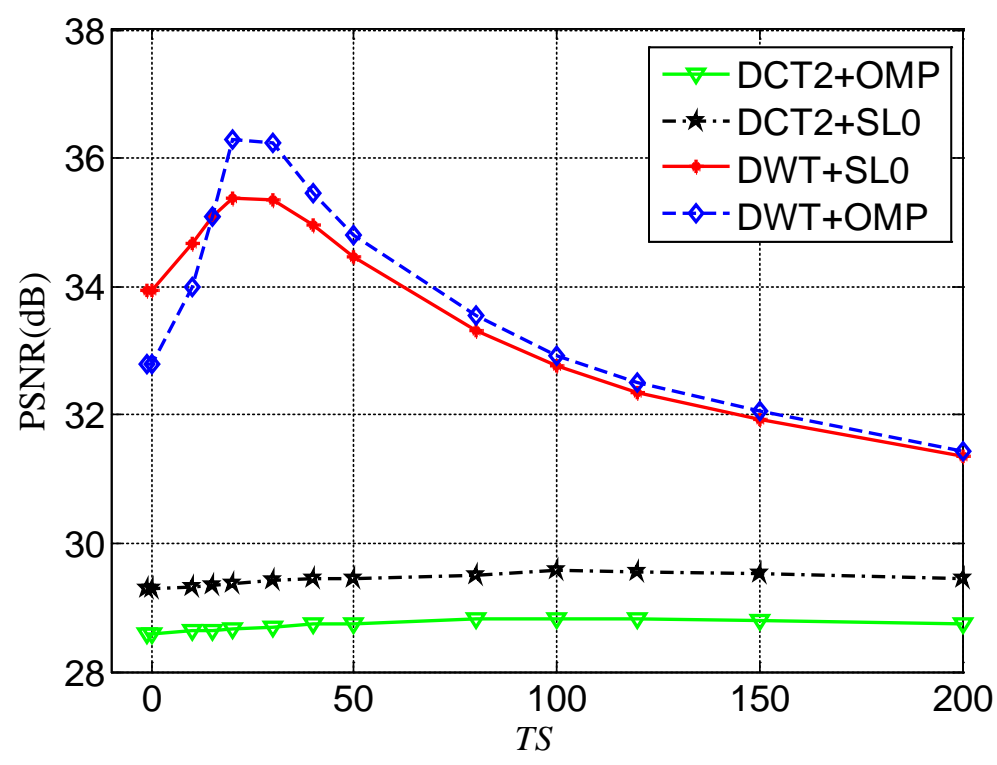

Fig.10 PSNR vs TS for Peppers with different sparsifying methods and reconstruction methods 


\subsection{The effect of the carrier image on simulation results}

Peppers is used as the original image, which is shown in Fig. 8(a), the carrier images are Goldhill, Brone, Aerial and Bridge with the same size, OMP is used in the reconstruction process of the CS, other simulation parameters are used the same as Section 4.1, and the corresponding encryption and decryption results are illustrated in Fig. 11. From Fig.11, it is clear that when the different carrier images are adopted, the different meaningful cipher images are obtained, which can effectively realize appearance security of the cipher image during transmission and store; and the most important is that the qualities of corresponding decrypted images are different, even the computation parameters are the same, which means the choosing of carrier images is important.

In order to quantitatively analyze the effect of carrier images on the quality of the decrypted images, we calculate the PSNR between the decrypted image and the plain image, when the threshold TS varies from 0 to 200, and the results are shown in Fig. 12. And here, TS=-1 means there is no sparse coefficient processing. From the figure, we come to the conclusion that firstly, when the carrier images are Brone or Bridge, the PSNR values are below $30 \mathrm{~dB}$ and they stay the same with the changing of TS; secondly, when Goldhill and Aerial image are chosen, the PSNR values are larger than $30 \mathrm{~dB}$, the maximum is $36.31 \mathrm{~dB}$ for Goldhill, and $35.92 \mathrm{~dB}$ for Aerial, the qualities of decrypted images are extraordinary; thirdly, TS has no negligible effect for Goldhill and Aerial, the PNSR values are the best around 20, and for Goldhill, they are changing from 36.31dB $(T S=20)$ to $31.43 \mathrm{~dB}(T S=200)$. 


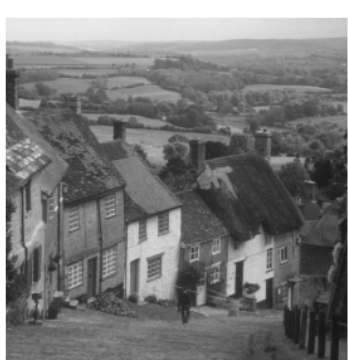

(a) Goldhill

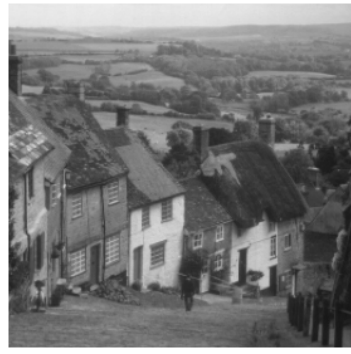

(e) Cipher image

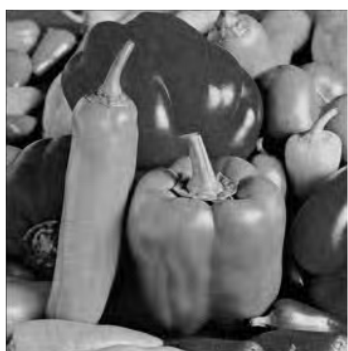

(i) Decrypted image

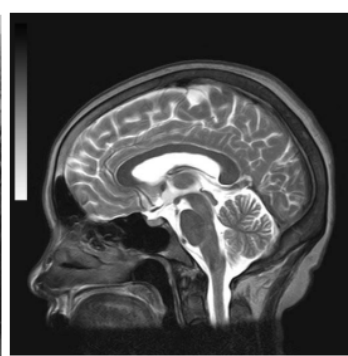

(b) Brone

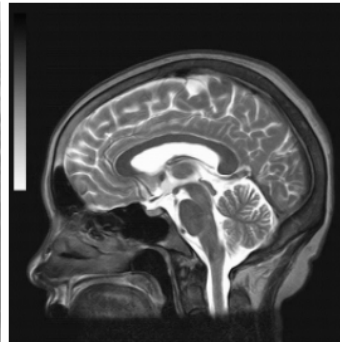

(f) Cipher image

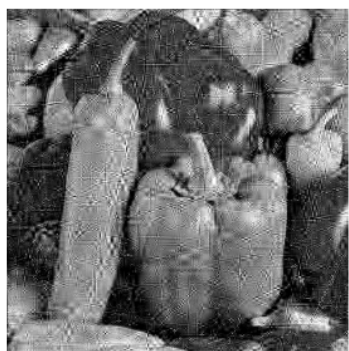

(j) Decrypted image

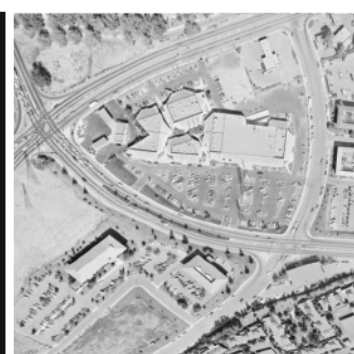

(c) Aerial

(d) Bridge

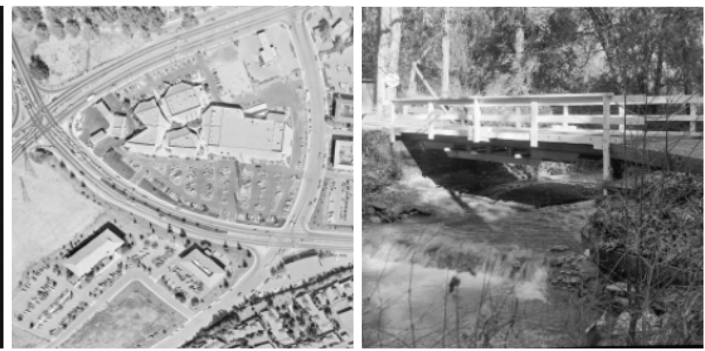

(g) Cipher image

(h) Cipher image

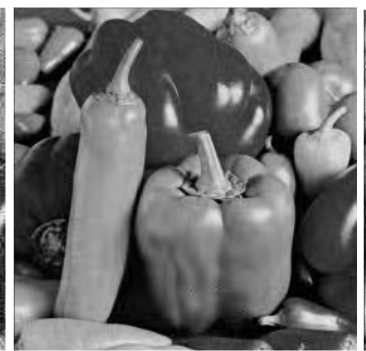

(k) Decrypted image

(l) Decrypted image

Fig.11 The encryption and decryption results for different carrier images

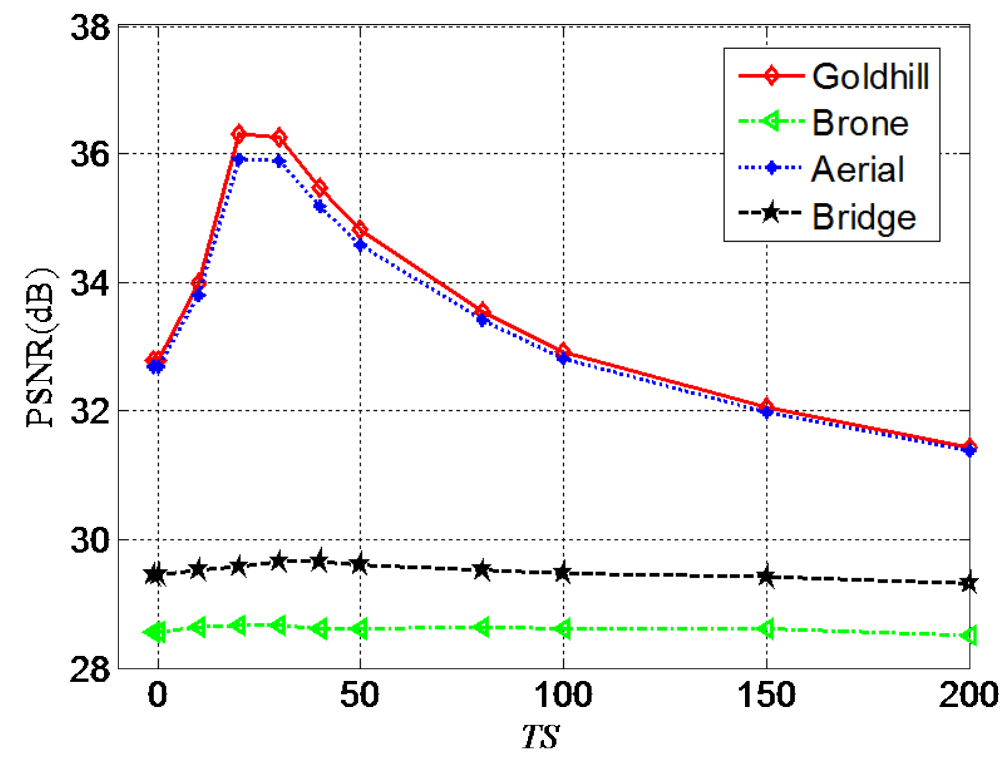

Fig.12 PSNR vs TS for Peppers with different carrier images 


\subsection{Simulation results of the compression and encryption method based on zigzag confusion}

\section{and CS}

In the above analyses, the carrier image and plain image have the same size, and compression ratio of the plain image is $C R=0.25$. If we remove Section 3.2.2 from the proposed encryption algorithm shown in Section 3.2, the rest Section 3.2.1 will be a good compression and encryption method based on zigzag confusion and CS, and next we analyze the performance of this method. In the simulation, the sparsifying method of the plain image makes use of DWT, the reconstruction method chooses OMP, threshold is set as $T S=50$, and other parameters are chosen as Section 4.1. Fig.13 is the encryption and decrypted results for Barbara $(512 \times 512)$ with different $C R$. As can be seen from the figure that when $C R$ varies from $0.25,0.4,0.6$ to 0.8 , the cipher images are compressed and noisy, nothing useful information can be found from them, the decoded image is just as the plain image from the appearance, and the proposed compression and encryption scheme can compress the plain image into the cipher image, reconstruct the original image, and has good encryption and decryption effect.

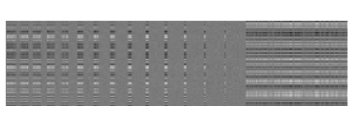

(a) Cipher image(0.25)

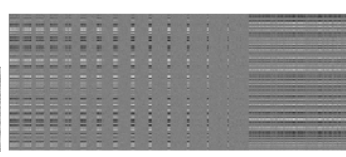

(b) Cipher image(0.4)

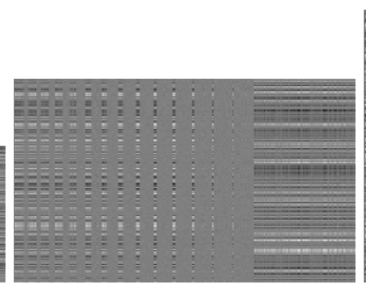

(c) Cipher image(0.6)

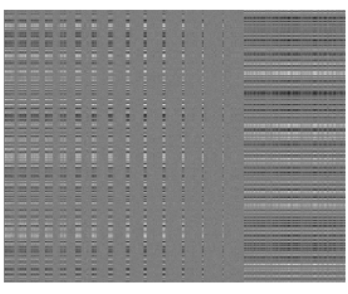

(d) Cipher image(0.8)
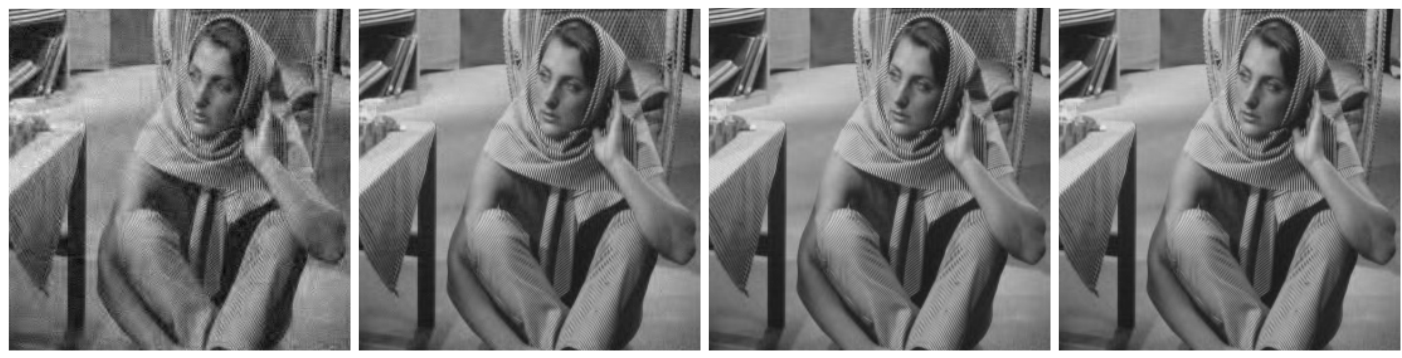

(e) Decoded image(0.25) (f) Decoded image(0.4) (g) Decoded image(0.6) (h) Decoded image(0.8)

Fig.13 Encryption and decryption results of Barbara $(512 \times 512)$ 
For four different plain images, the relationship of PSNR and $C R$ is shown in Fig.14. From the figure, it is clear that (1) with the increasing of $C R$, more data are sampled for reconstruction, thus the PSNR is larger, and the reconstruction quality is better; (2) when the $C R$ is fixed, different images have different PSNR and construction quality, and this has something to do with the sparsifying methods and reconstruction methods; (3) when $C R=0.25$, which means $25 \%$ of data is sampled for reconstructing the plain image, the PSNR values of Lena and Peppers are above 30dB, and the reconstructed images have extraordinary good appearance effect. In other words, when we set the reconstruction requirements as PSNR=30dB, we must sample about $80 \%$ of data and $40 \%$ of data for Baboon and Barbara, respectively, but for Lena and Peppers, we can pick out 25\% of data to meet the requirements.

To conclude, the proposed compression and encryption method based on zigzag confusion and CS can encrypt and compress the plain image effectively.

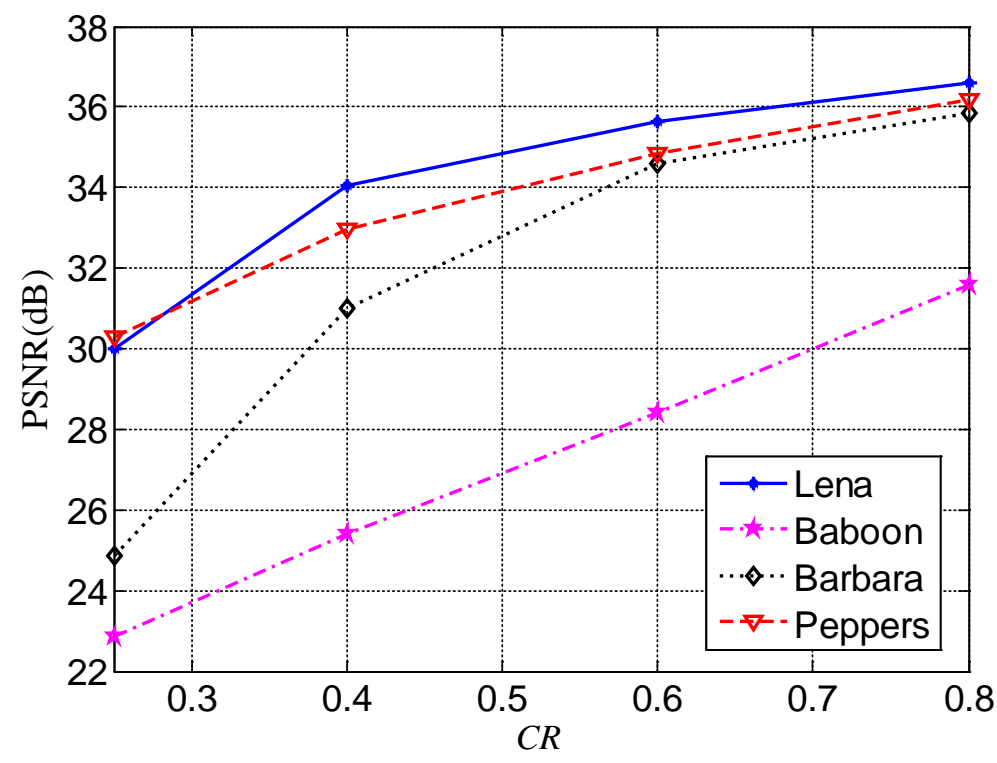

Fig.14 PSNR vs CR for different plain images

\section{Performance analyses}

\subsection{Key space analysis}


The secret key of the proposed encryption algorithm mainly includes that: (1) the 256-bit hash value $K$ obtained from the SHA 256 hash function of the plain image; (2) the given parameters: $x_{0}^{\prime}, y_{0}^{\prime}, r^{\prime}, z_{0}^{\prime}$. Besides, the threshold $T S$, and at least 37 types of existing wavelet filters of DWT can also be taken as secret key. If the computation precision of the computer is $10^{-14}$, and then the key space is about $37^{2} \times 2^{296}$.

In addition, in our encryption scheme, the carrier image is used as a visual protection to the plain images. Different carrier images can produce different visually secure cipher images. Therefore, the key space of our method is large enough to resist all kinds of brute-force attacks.

\subsection{Key sensitivity analysis}

A good image encryption algorithm should have a high sensitivity to secret key in encryption and decryption process. The Pirate $(512 \times 512)$ is used as the plain image, and the carrier image is Lena (512×512). In encryption process, we change each of $x_{0}^{\prime}, y_{0}^{\prime}, r^{\prime}, z_{0}^{\prime}$ by adding $10^{-14}$, we modify a bit of 256-bit hash value $K$, and a new $K 1$ is obtained, and we change any one pixel of the plain image, a completely different hash value $K 2$ is gotten. These changed secret key is used to encrypt the original image and every time one parameter is changed and others are constant. $K$, $K 1$ and $K 2$ are shown as follows:

$K=$ 2a9cb66cd4eaf51426b32d31aa84d695ae9be42bbff7222f9aa7275ef271c002

K1= 2a9cb66cd4eaf51426b32d31aa84d695ae9be42bbff7222f9aa7275ef271c003

$K 2=$ f064f43ced44f61d4c5fa1175838dfb1fa454e944ba72165009710d21749ff1e

The key sensitivity results in encryption process are shown in Fig.15. In Fig.15, (a) is the plain image, (b) is the carrier image, (c) is the visually secure cipher image with the right key, (e)-(g) are their respective histograms; (d), (i)-(l), (q) are the encrypted image with the changed 
parameters, and (h), (m)-(p), (r) are their histograms, respectively. As can be seen from Fig.15 that when the key is changed, the corresponding cipher image is just as the carrier image from the appearance, but the corresponding histogram has a little difference compared with that of the carrier image. That is because the carrier image plays an important role in the visually secure cipher images, and thus the modified key has a small effect on them.

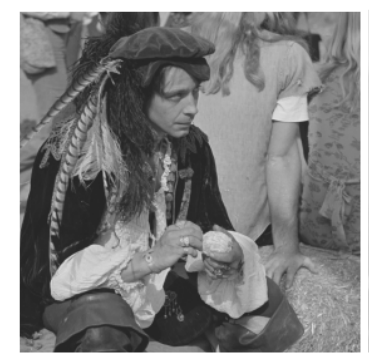

(a) Plain image Pirate

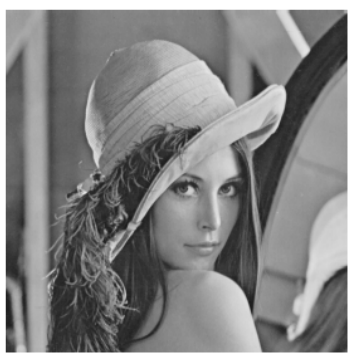

(b) Carrier image

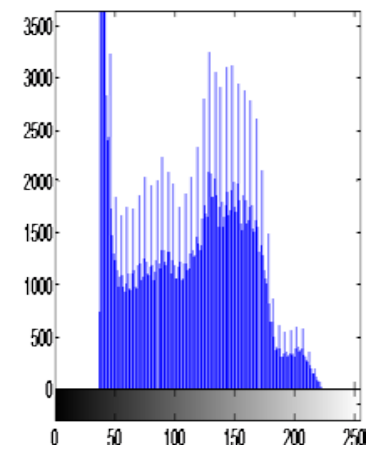

(e) Histogram of (a)

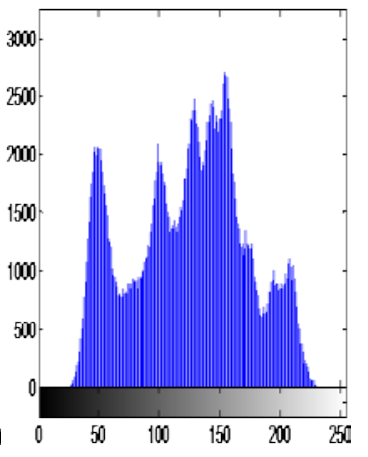

(f) Histogram of (b)

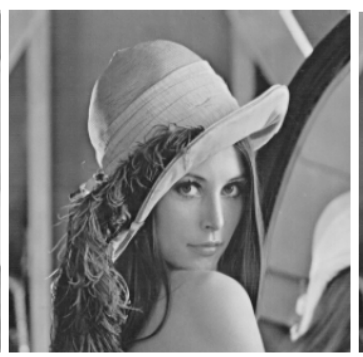

(c) The right key

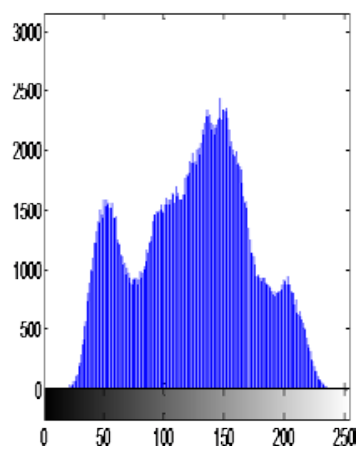

(g) Histogram of (c)

(h) Histogram of (d)

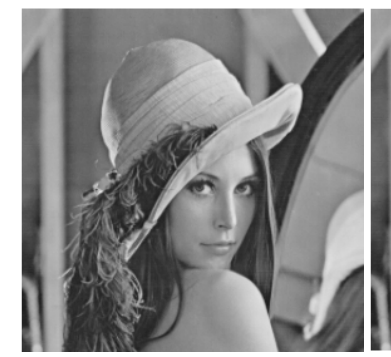

(i) $y_{0}^{\prime}+10^{-14}$

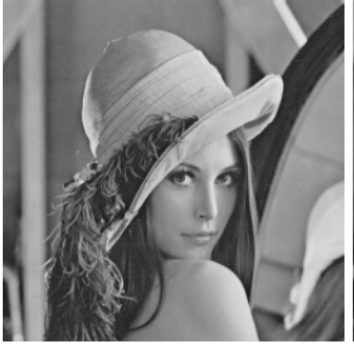

(j) $r^{\prime}+10^{-14}$

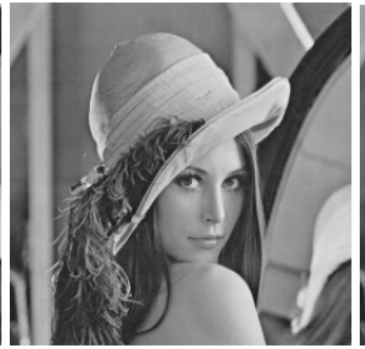

(k) $z_{0}^{\prime}+10^{-14}$

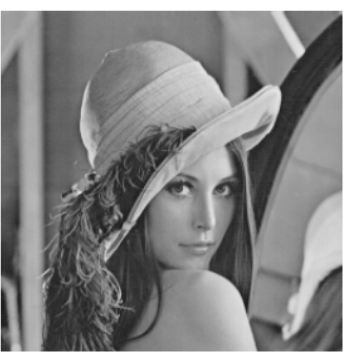

(d) $x_{0}^{\prime}+10^{-14}$
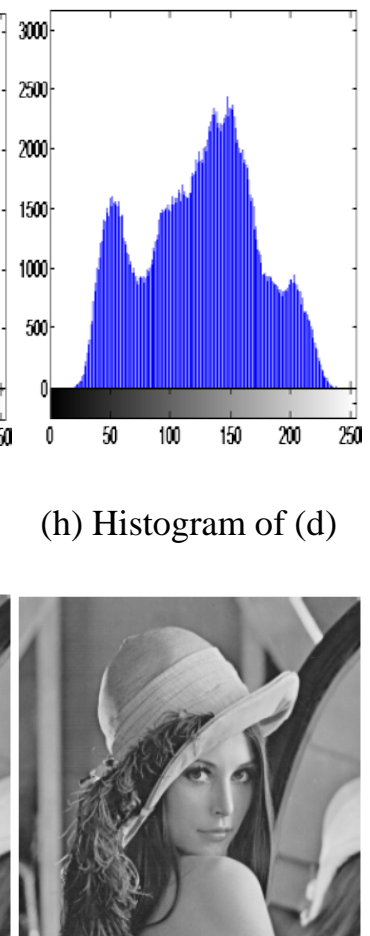

(l) $K 1$ 


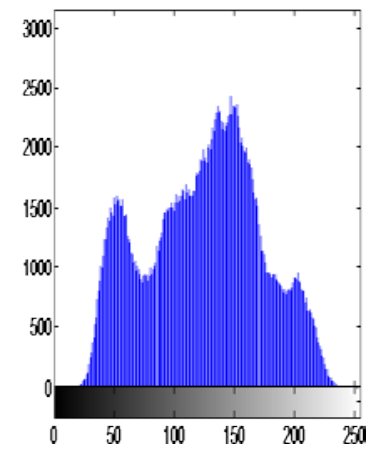

(m) Histogram of (i)

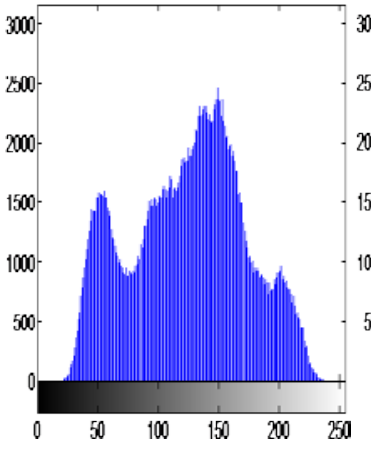

(n) Histogram of (j)

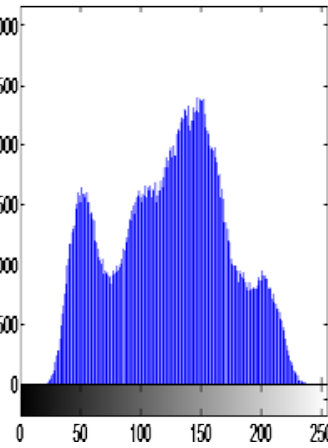

(o) Histogram of (k)

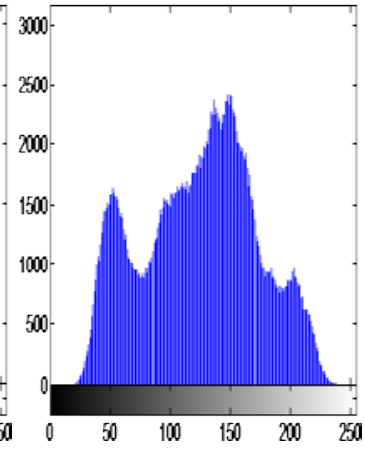

(p) Histogram of (l)

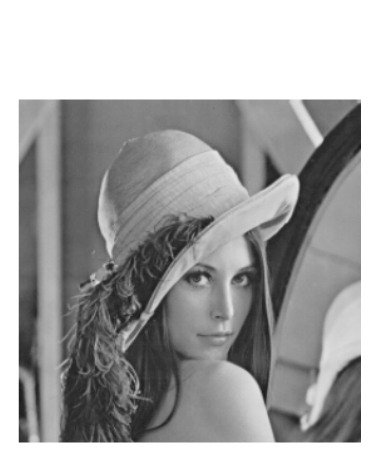

(q) $K 2$

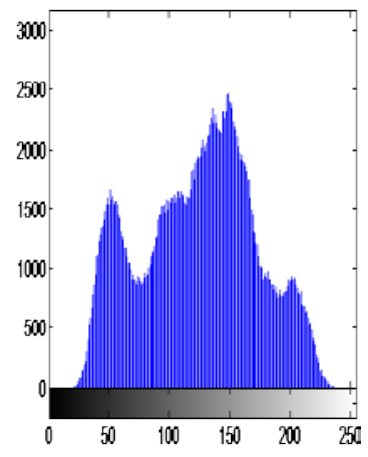

(r) Histogram of (q)

Fig.15 Key sensitivity results in encryption process

Next, we use the modified keys as above to decrypt the cipher image (Fig.15(c)) to test the key sensitivity in the decryption process. The results are shown in Fig.16, (a)-(d), (i)-(k) are the decrypted image with different keys, and (e)-(h), (l)-(n) are their respective histograms. It is evident that the reconstructed image can be recovered with the right key, and it is just the same with the plain image Fig.15(a), and the PSNR is 32.26dB; when the key has a trivial change, the noisy decrypted images can be obtained, and nothing useful information can be gotten from them.

Therefore, the proposed encryption scheme is highly sensitive to the key in the encryption and decryption process. 

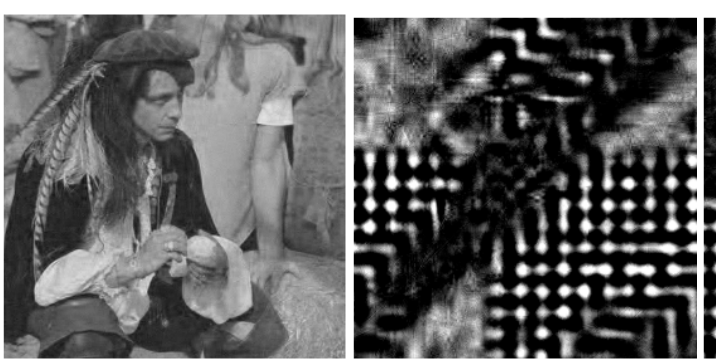

(a) The right key

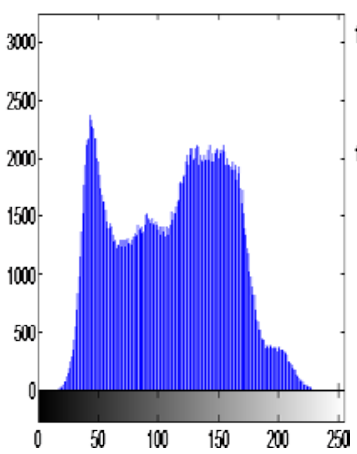

(b) $x_{0}^{\prime}+10^{-14}$

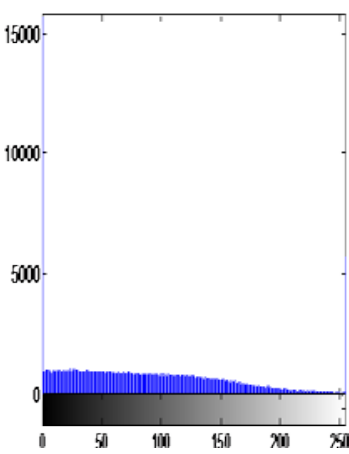

(e) Histogram of (a)

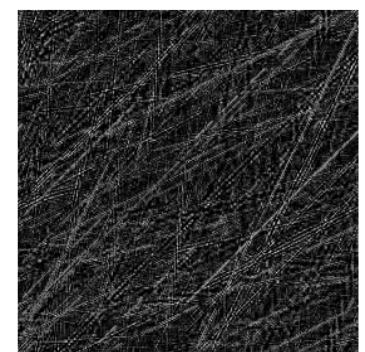

(i) $z_{0}^{\prime}+10^{-14}$ (f) Histogram of (b)

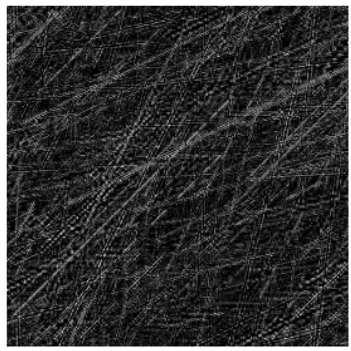

(j) $K 1$

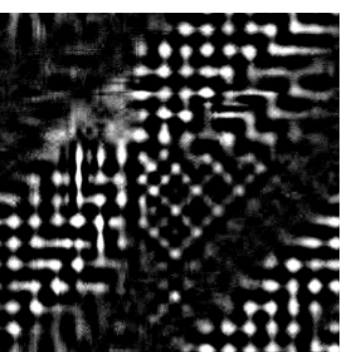

(c) $y_{0}^{\prime}+10^{-14}$

(d) $r^{\prime}+10^{-14}$

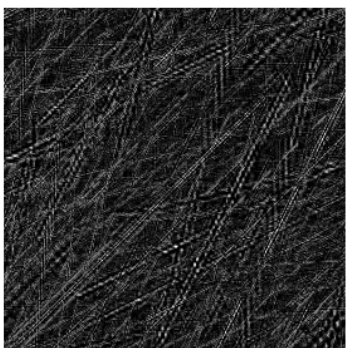

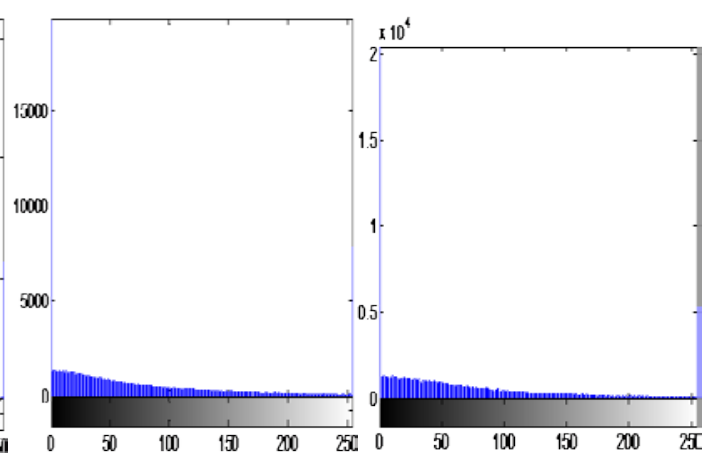

(g) Histogram of (c)

(h) Histogram of (d)

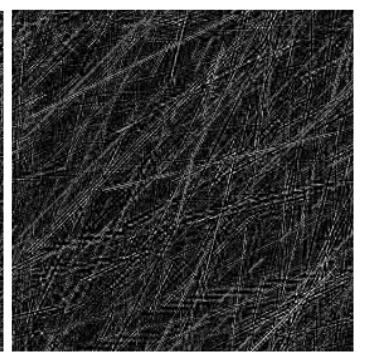

(k) $K 2$
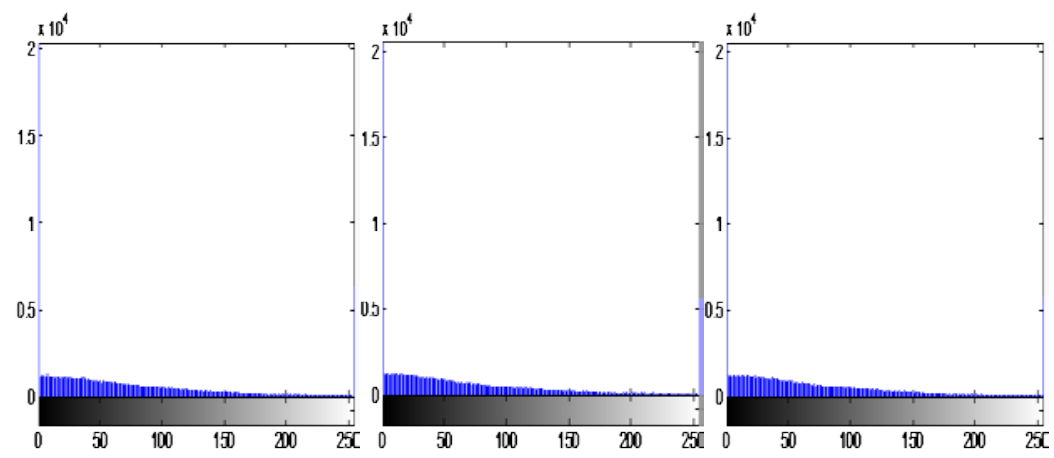

(l) Histogram of (i)

(m) Histogram of (j)

(n) Histogram of (k)

Fig.16 Key sensitivity analyses in decryption process

\subsection{Robustness analysis}




\subsubsection{Noise attack}

During the transmission, the visually secure cipher images are easily affected by all kinds of noises, such as Gaussian noise (GN), Salt \& Pepper noise (SPN) and Speckle noise (SN). The noise makes recovering the plain image more difficult. And an image encryption algorithm with the ability of resisting noise attack is more suitable for real applications. Next, we will measure the ability of the proposed algorithm to resist the noise attack. In the simulation, Barbara $(512 \times 512)$ is used as the plain image and shown in Fig.8(b), the same size Lena (Fig.6(a)) is the carrier image, and other parameters are set as Section 4.1. The visually secure cipher image is shown in Fig.17(a), and it is contaminated by noises, the noise intensities are $0.0001 \%, 0.0003 \%, 0.0005 \%, 0.0007 \%$, and the noisy cipher images and the corresponding decrypted images are shown in Fig.17. In Fig.17, apart from the last row, the first, third, fifth rows are all cipher images with different types of noise, and the second, fourth, sixth rows are the decrypted image, respectively.

The PSNR between the decrypted image and the plain image is calculated and shown in Fig.18. From them, we can see that (1) GN noise has the largest effect on the decryption process, the PSNR values vary from $30.30 \mathrm{~dB}$ to $28.42 \mathrm{~dB}$, when the noise intensities change from $0.0001 \%$ to $0.0007 \%$, the quality of decrypted images decrease with the increasing of the noise intensity, and they are all recognized visually; (2) Our encryption scheme has the strongest resisting capability to SPN, the PSNR values are almost the same during the whole process, and they are changing from $32.60 \mathrm{~dB}$ to $32.35 \mathrm{~dB}$, the decrypted images have the best visual appearance; (3) The proposed encryption algorithm has a certain withstanding ability to SN, the PSNR values vary from $32.60 \mathrm{~dB}$ to $29.23 \mathrm{~dB}$, and the corresponding recovered images are also meaningful. In a word, in the presence of noise, the recovered images are shown clearly, and the proposed encryption 
scheme has good robustness to noise attacks.

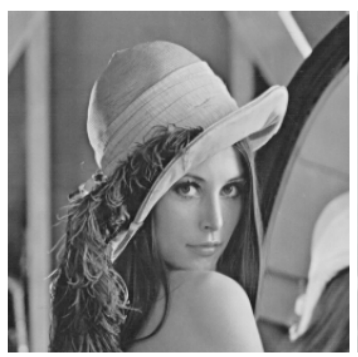

(a) Cipher image no noise

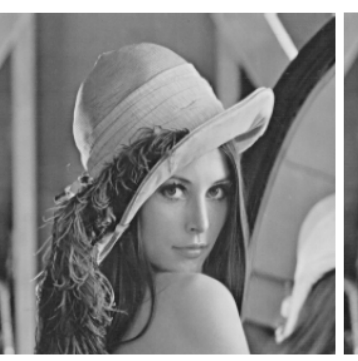

(b) $0.0001 \% \mathrm{GN}$

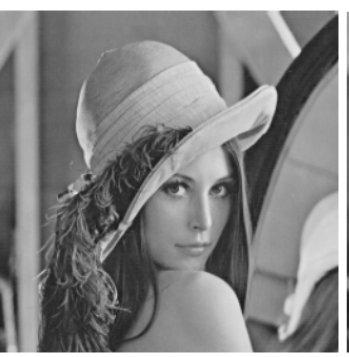

(c) $0.0003 \% \mathrm{GN}$

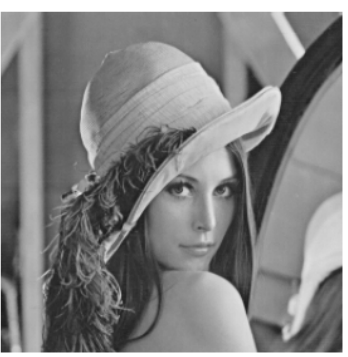

(d) $0.0005 \% \mathrm{GN}$

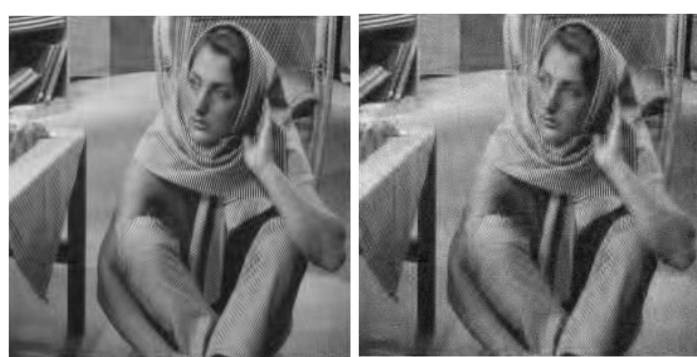

(e) Decrypted image no noise (f) $0.0001 \%$ GN

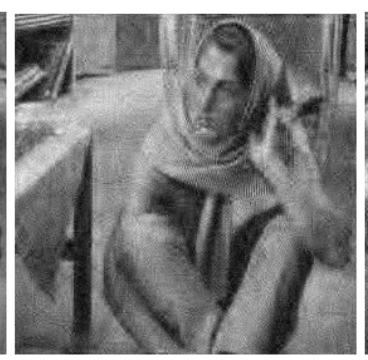

(g) $0.0003 \% \mathrm{GN}$

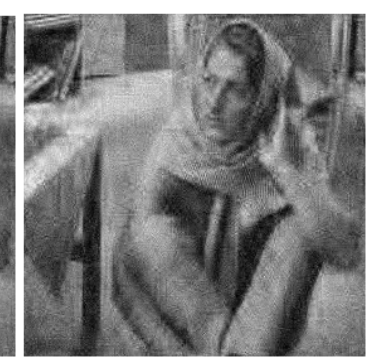

(h) $0.0005 \% \mathrm{GN}$

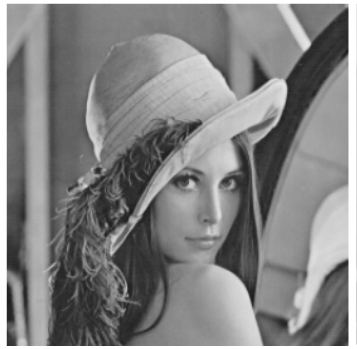

(i) $0.0007 \% \mathrm{GN}$

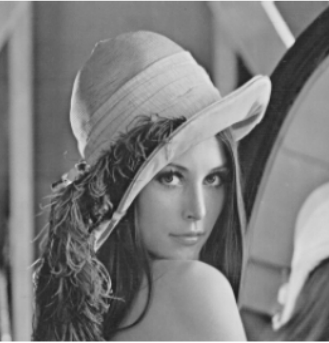

(j) $0.0001 \% \mathrm{SPN}$

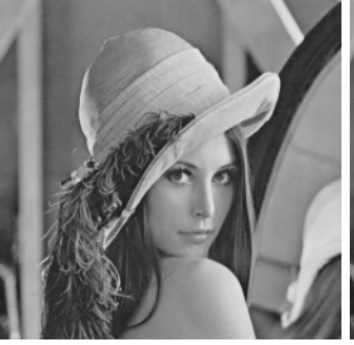

(k) $0.0003 \% \mathrm{SPN}$

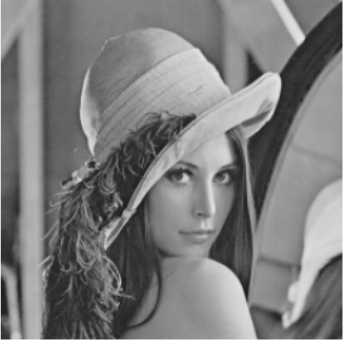

(l) $0.0005 \% \mathrm{SPN}$

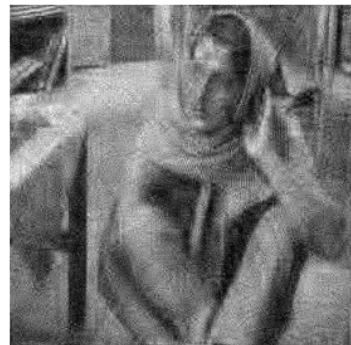

(m) $0.0007 \% \mathrm{GN}$

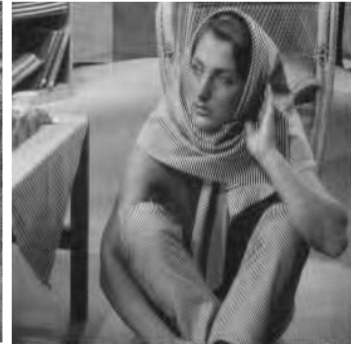

(n) $0.0001 \% \mathrm{SPN}$

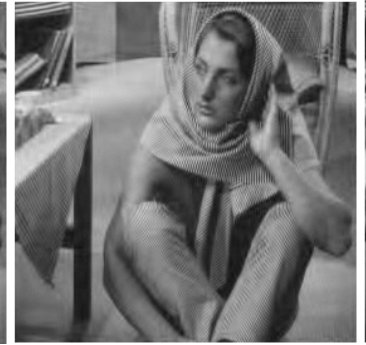

(o) $0.0003 \% \mathrm{SPN}$

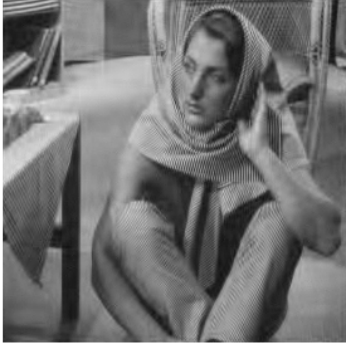

(p) $0.0005 \% \mathrm{SPN}$

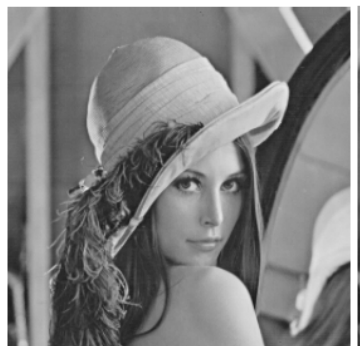

(q) $0.0007 \% \mathrm{SPN}$

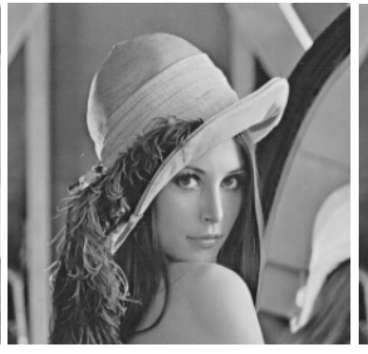

(r) $0.0001 \% \mathrm{SN}$

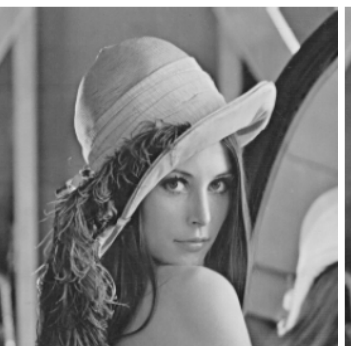

(s) $0.0003 \% \mathrm{SN}$

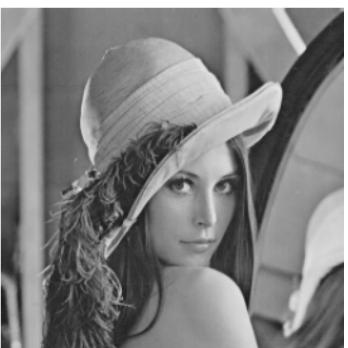

(t) $0.0005 \% \mathrm{SN}$ 


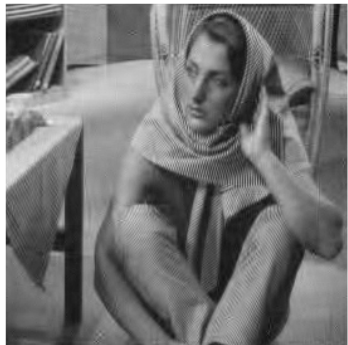

(u) $0.0007 \% \mathrm{SPN}$

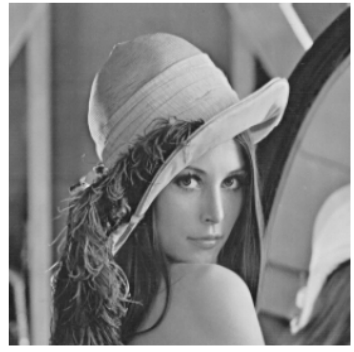

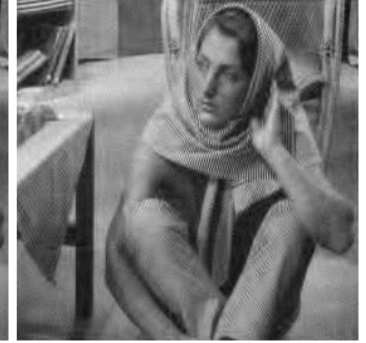

(w) $0.0003 \% \mathrm{SN}$

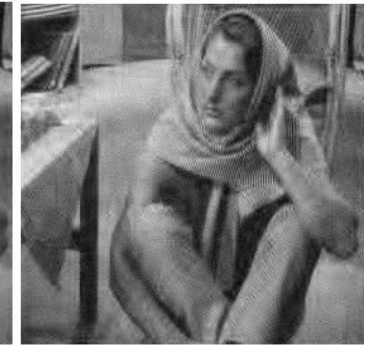

(x) $0.0005 \% \mathrm{SN}$

(y)Cipher image with $0.0007 \%$ SN (z)Decrypted image of (y)

Fig.17 The simulation results with noise attacks

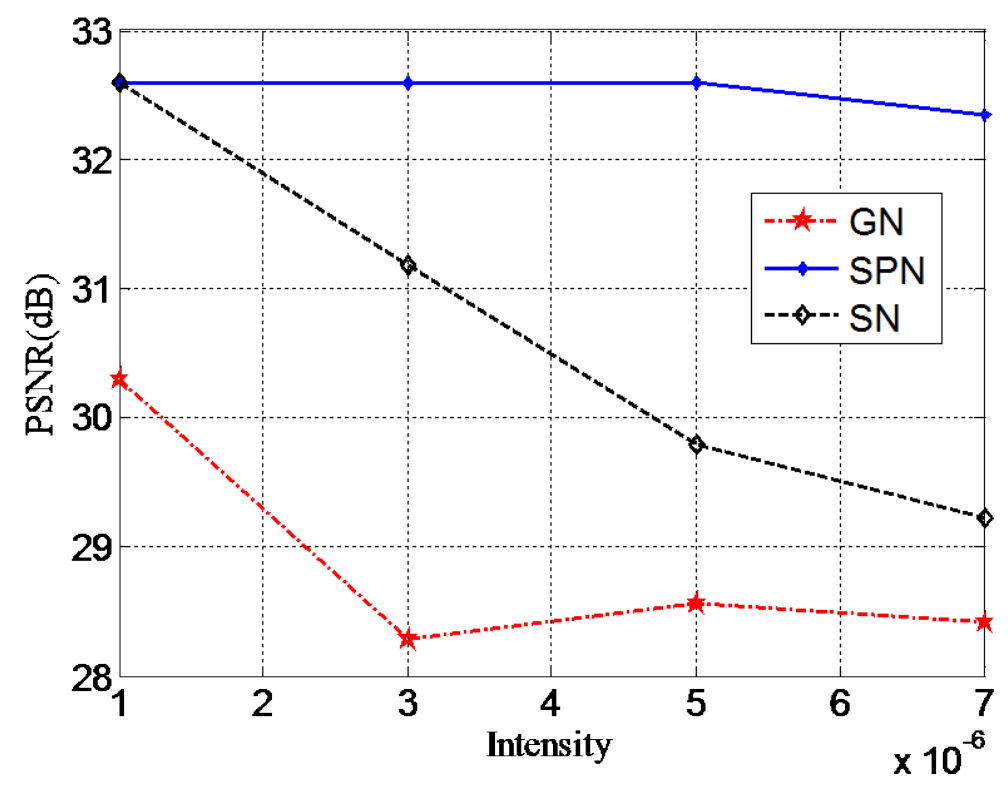

Fig.18 PSNR between the decrypted image and the plain image with different noise

\subsubsection{Occlusion attack}

The robustness of a cryptosystem against occlusion is also an important requirement in the real image communication. The data loss of the cipher image can affect the recovered images greatly. The encrypted image (shown in Fig.17(a)) with four different data losses are shown in Fig. 19(a), (b), (c), (d) and the corresponding recovered images are illustrated in Fig.19 (e), (f) , (g) and 
(h), respectively. As can be seen from Fig.19 that when the data loss size is varying from 32×32 to $112 \times 112$, the quality of recovered image decreases, and PSNR values are changing from $29.74 \mathrm{~dB}$ to $28.02 \mathrm{~dB}$, and our scheme could resist data loss attack to a certain degree, since there is $25 \%$ of data sampled from the plain image, the data is few such that more data loss can add more difficulties in recovering the original image.

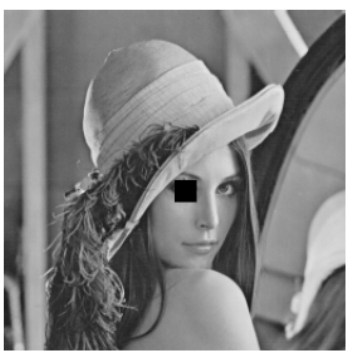

(a) $32 \times 32$ data loss

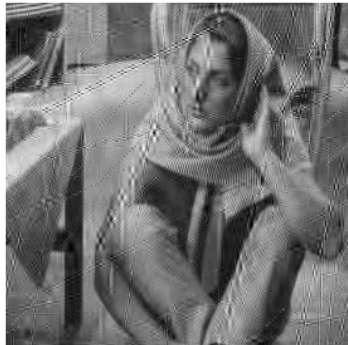

(e) Decrypted image
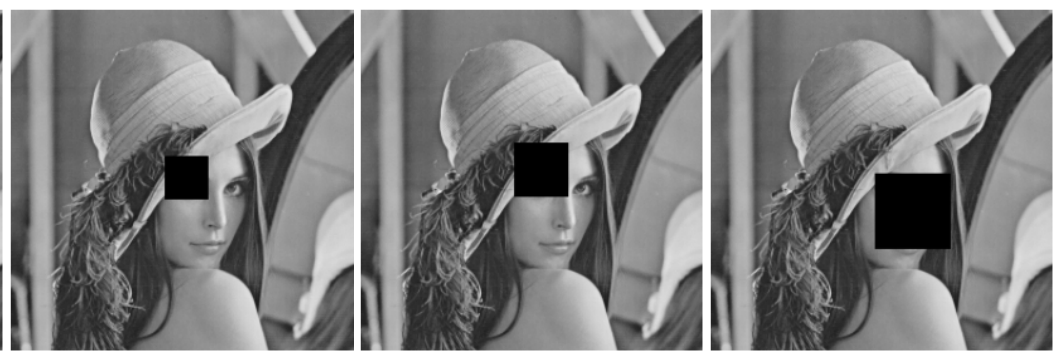

(b) $64 \times 64$ data loss

(c) $80 \times 80$ data loss

(d) $112 \times 112$ data loss
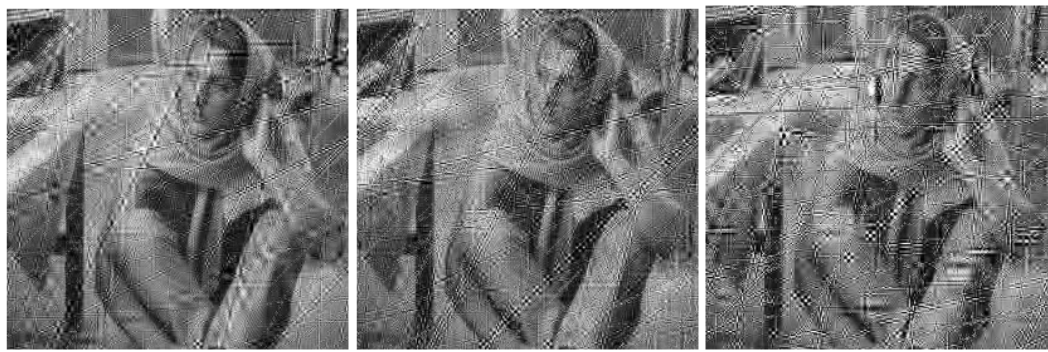

(h) Decrypted image

Fig.19 Occlusion attack results

\subsection{Known-plaintext and chosen-plaintext attacks}

In the paper, some methods are employed to improve the resistance of the proposed encryption algorithm to known-plaintext and chosen-plaintext attacks. Firstly, SHA 256 hash function of the original image is computed, next the parameters used in the zigzag confusion process and the production of the chaos-based measurement matrix are decided by the plain image. Thus, when different plain images are encrypted, the corresponding key stream changes too, and the different cipher images are obtained. The hackers cannot get useful information by encrypting 
some special images since the resultant information depends on the original images. Therefore, the proposed scheme can well withstand the known-plaintext and chosen-plaintext attacks.

\subsection{Time complexity analysis}

Time complexity is also an important index to evaluate the performance of the encryption scheme. The encryption process consists of compression and encryption of the plain image, and embedding process, and the decryption one is composed of extraction process of the compressed cipher image and reconstruction process of the plain image. The execution times for different images are listed in Table 1.

From Table 1, we can watch that firstly, in the total encryption and decryption times, embedding and extraction times of the compressed cipher image are very little, in detail, in encryption process, compression and encryption of the plain image accounts for about $60 \%$ of the total time, and in decryption process, the reconstruction process costs around $95 \%$ of the total time; secondly, when the image sizes vary from $256 \times 256$ to $1024 \times 1024$, the encryption time is from 0.4049s to $3.9024 s$, but the decryption time is from 1.4724 s to 122.4369 s. Thus, the proposed encryption scheme is suitable for the small and medium size images, when the size of the image is larger, the time complexity is very higher. In the following work, we will plan to substitute block compressive sensing (BCS) for compressive sensing (CS) to reduce the computation complexity and shorten the encryption and decryption time. 
Table 1 Encryption and decryption time for different images (time unit: s)

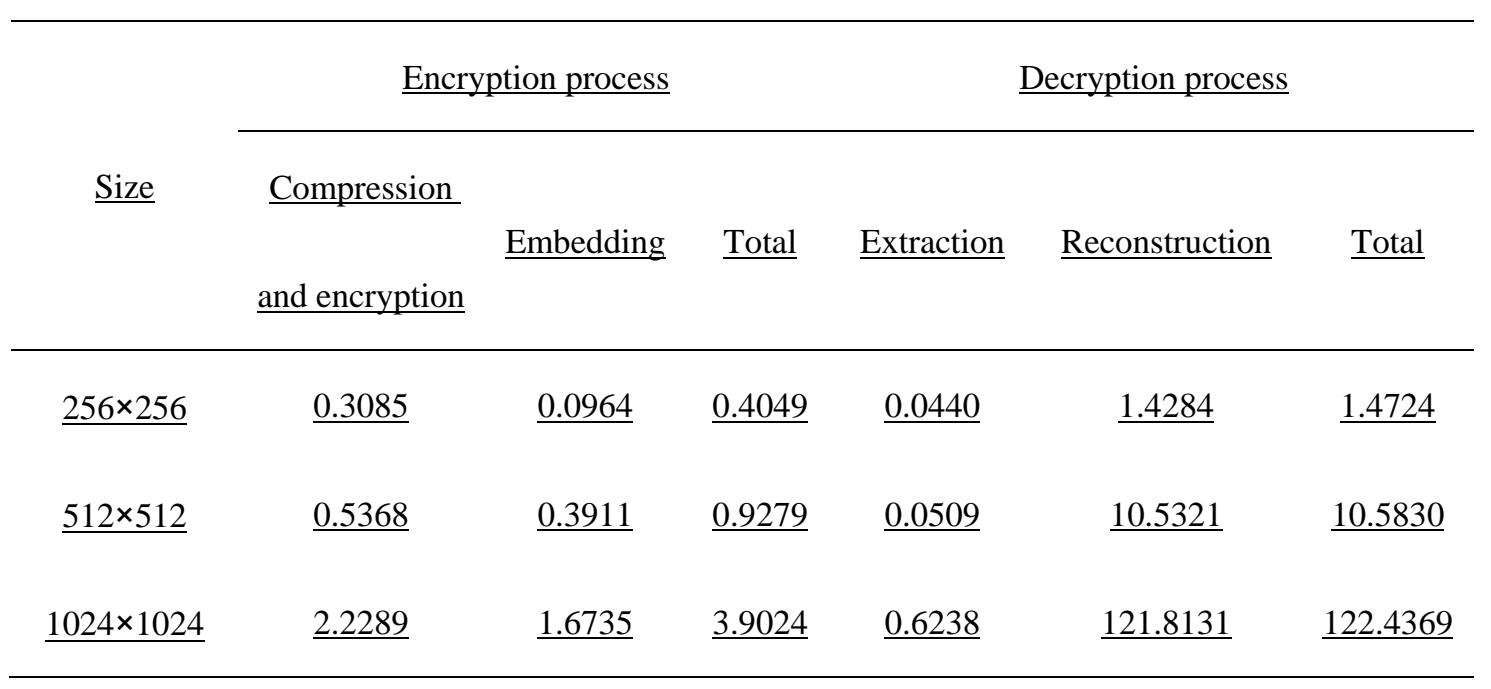

\subsection{Comparison with other encryption algorithms}

The existing image encryption scheme mostly turns a meaningful plain image into a meaningless cipher image, and the noise-like or texture-like cipher image is transmitted and stored. Additionally, some image encryption algorithms based on compressive sensing may encrypt the gray scale images [34-36] or color images [37], but the cipher images are all noisy. When these cipher images are transmitted, they are easily found and attacked, thus the appearance security of the original images cannot be realized. Compared with these kinds of image encryption algorithms, our scheme embeds the cipher image into a carrier image after the plain image is encrypted, the final obtained cipher image is meaningful, it has a secure appearance to avoid being found and attacked, and the image data security and appearance security can be attained at the same time, and our proposed algorithm is more secure.

Zhou et al [48] proposed a novel image encryption algorithm. Firstly, the plain image of $m \times n$ is encrypted into a cipher image, next the $m \times n$ cipher image is embedded into a reference image of $2 m \times 2 n$, and the final encrypted image is meaningful, but its size is $2 m \times 2 n$, and is four times of the plain image. The sharp increasing size of the cipher image needs more encryption time, 
transmission bandwidth and storage. However, for our encryption scheme, the zigzag confusion dependence on the plain image is used to encrypt the plain image and upgrade the reconstruction effect, compressive sensing is employed to compress and encrypt the original image, and then the compressed cipher image is embedded into a carrier image, the embedded method is different for different carrier image, and a visually secure cipher image is gotten. In addition, the size of the cipher image and the plain image is equal, and it is more efficient from these points. In the future, with the improvement of compression performance of compressive sensing, the size of the carrier image and cipher image may be smaller, and then our algorithm should be better and more efficient.

\section{Conclusions}

In the paper, a visually secure image encryption scheme based on compressive sensing is introduced. The plain image is firstly transformed to the coefficient matrix using DWT, and then scrambled by a plain image related zigzag path and encrypted into a compressed cipher image by compressive sensing. Secondly, the cipher image is embedded into a carrier image, and we finally get a visually secure cipher image. Compared with the existing encryption algorithms, the proposed encryption scheme has three advantages. Firstly, it has high security level, it can achieve image data security and appearance security simultaneously; secondly, the final cipher image has the same size with the plain image, it can transmits and stores fast over the Internet, and there is no additional transmission bandwidth and memory; and lastly, the encryption scheme has high sensitivity to the plain image, the parameters used in the zigzag confusion process and the generation of the chaos-based measurement matrix are computed by the SHA 256 hash function of 
the original image, our algorithm can withstand the known-plaintext and chosen-plaintext attacks effectively.

Simulation results and security analyses demonstrate that the proposed encryption scheme has large key space, high key sensitivity, good robustness to noise and occlusion attack, and it can applied in the image secure communication. In the future, we will replace compressive sensing (CS) with block compressive sensing (BCS) to improve the encryption and decryption speed and shorten the execution time, and then the cryptosystem is more efficient.

\section{Acknowledgments}

All the authors are deeply grateful to the editors for smooth and fast handling of the manuscript. The authors would also like to thank the anonymous referees for their valuable suggestions to improve the quality of this paper. This work is supported by the National Natural Science Foundation of China (Grant No. 61203094 and 61305042), National Science Foundation of the United States (Grant No. CNS-1253424 and ECCS-1202225), Science and Technology Foundation of Henan Province of China (Grant No. 152102210048), Foundation and Frontier Project of Henan Province of China(Grant No. 162300410196), Natural Science Foundation of Educational Committee of Henan Province of China (Grant No. 14A413015) and the Research Foundation of Henan University (Grant No. xxjc20140006).

\section{References}

[1] Akram Belazi, Ahmed A Abd El-Latif, Safya Belghith. A novel image encryption scheme based on substitution-permutation network and chaos. Signal Process. 2016, 128: 155-170 
[2] H J Liu, Abdurahman Kadir. Asymmetric color image encryption scheme using 2D discrete-time map. Signal Process. 2015, 113: 104-112

[3] W Zhang, H Yu, Y L Zhao, Z L Zhu. Image encryption based on three-dimensional bit matrix permutation. Signal Process. 2016, 118: 36-50.

[4] Y C Zhou, L Bao, Philip C L Chen. A new 1D chaotic system for image encryption. Signal Process. 2014, 97: 172-182.

[5] Seyed Mohammad Seyedzadeh, Benyamin Norouzi, Mohammad Reza Mosavi, Sattar Mirzakuchaki. A novel color image encryption algorithm based on spatial permutation and quantum chaotic map. Nonlinear Dyn. 2015, 81: 511-529.

[6] Manish Kumar, Akhlad Iqbal, Pranjal Kumar. A new RGB image encryption algorithm based on DNA encoding and elliptic curve Diffie-Hellman cryptography. Signal Process. 2016, 125: $187-202$

[7] X Y Wang, Y Q Zhang, X M Bao. A novel chaotic image encryption scheme using DNA sequence operations. Opt Lasers Eng. 2015, 73: 53-61.

[8] R Guesmi, M A B Farah, A Kachouri, M Samet. A novel chaos-based image encryption using DNA sequence operation and secure hash algorithm SHA-2. Nonlinear Dyn. 2016, 83:1123-1136. [9] Rasul Enayatifar, Hossein Javedani Sadaei, Abdul Hanan Abdullah, Malrey Lee, Ismail Fauzi Isnin. A novel chaotic based image encryption using a hybrid model of deoxyribonucleic acid and cellular automata. Opt Laser Eng. 2015, 71:33-41.

[10] Amina Souyah, Kamel Mohamed Faraoun. Fast and efficient randomized encryption scheme for digital images based on Quadtree decomposition and reversible memory cellular automata. Nonlinear Dyn. 2016, 84: 715-732. 
[11] P Ping, F Xu, Z J Wang. Image encryption based on non-affine and balanced cellular automata. Signal Process. 2014, 105: 419-429.

[12] W Chen. Optical multiple-image encryption using three-dimensional space. IEEE Photonics J. 2016, 8: 6900608

[13] W Chen. Single-shot imaging without reference wave using binary intensity pattern for optically-secured-based correlation. IEEE Photonics J. 2016, 8: 6900209.

[14] Y Wang, C Quan, C J Tay. Asymmetric optical image encryption based on an improved amplitude-phase retrieval algorithm. Opt Laser Eng. 2016, 78: 8-16.

[15] X L Chai. An image encryption algorithm based on bit level Brownian motion and new chaotic systems. Multimed Tools Appl. 2015, doi: 10.1007/ s11042-015-3088-1.

[16] X Y Wang, D H Xu. A novel image encryption scheme based on Brownian motion and PWLCM chaotic system. Nonlinear Dyn. 2014, 75(1-2): 345-353.

[17] G D Ye. A block image encryption algorithm based on wave transmission and chaotic systems. Nonlinear Dyn. 2014, 75: 417-427.

[18] X F Liao, S Y Lai, Q Zhou. A novel image encryption algorithm based on self-adaptive wave transmission. Signal Process. 2010, 90: 2714-2722.

[19] Y Wu, Y C Zhou, P Noonan Joseph, Agaian Sos. Design of image cipher using latin squares. Inf Sci. 2014, 264:317-339.

[20] Y Liu, J Tang, T Xie. Cryptanalyzing a RGB image encryption algorithm based on DNA encoding and chaos map. Opt Lasers Eng. 2014, 60: 111-115.

[21] Y S Zhang, D Xiao. On the security of symmetric ciphers based on DNA coding. Inf Sci. 2014, 28: 254-261. 
[22] Q Zhang, L Liu, X Wei. Improved algorithm for image encryption based on DNA encoding and multi-chaotic maps. AEU-Int J Electron C. 2014, 68: 186-192.

[23] C Q Li. Cracking a hierarchical chaotic image encryption algorithm based on permutation. Signal Process. 2016, 118: 203-210.

[24] Wun-she Yap, Raphael C W Phan, Wei-chuen Yau, Swee-Huay Heng. Cryptanalysis of a new image alternate encryption algorithm based on chaotic map. Nonlinear Dyn. 2015, 80: 1483-1491.

[25] Y S Zhang, D Xiao. Cryptanalysis of S-box-only chaotic image ciphers against chosen plaintext attack. Nonlinear Dyn. 2013, 72: 751-756.

[26] C X Zhu, S Y Xu, Y P Hu, et al. Breaking a novel image encryption scheme based on Brownian motion and PWLCM chaotic system. Nonlinear Dyn. 2015, 79(2):1511-1518.

[27] J X Chen, Z L Zhu, C Fu, L B Zhang, H Yu. Analysis and improvement of a double-image encryption scheme using pixel scrambling technique in gyrator domains. Opt Lasers Eng. 2015, 66: $1-9$.

[28] C Q Li, Y S Liu, T Xie, Michael Z Q Chen. Breaking a novel image encryption scheme based on improved hyperchaotic sequences. Nonlinear Dyn. 2013, 73: 2083-2089.

[29] X Wang, G He. Cryptanalysis on a novel image encryption method based on total shuffling scheme. Opt Commun. 2011, 284 (24):5804-5807.

[30] A Akhavan, A Samsudin, A Akhshani. Cryptanalysis of "an improvement over an image encryption method based on total shuffling”. Opt Commun. 2015, 350: 77-82.

[31] X Y Wang, L T Liu. Cryptanalysis of a parallel sub-image encryption method with high-dimensional chaos. Nonlinear Dyn. 2013, 73: 795-800.

[32] W Y Wen, Y S Zhang, Y M Fang, Z J Fang. Image salient regions encryption for generating 
visually meaningful ciphertext image. Neural Comput \& Applic. 2016, Doi: $10.1007 / \mathrm{s} 00521-016-2490-6$

[33] Y S Zhang, Leo Y Zhang, J T Zhou, L C Liu, F Chen, X He. A review of compressive sensing in information security field. IEEE Access. 2016, 5: 2507-2519.

[34] H Fang, S A Vorobyov, H Jiang, O Taheri. Permutation meets parallel compressed sensing: How to relax restricted isometry property for 2D sparse signals. IEEE T Signal Process. 2014, 62:196-210.

[35] Y S Zhang, J T Zhou, F Chen, Leo Y Zhang, Wong Kwok-Wo, XHe, D Xiao. Embedding cryptographic features in compressive sensing. Neurocomputing. 2016, 205: 472-480.

[36] N R Zhou, H L Li, D Wang, S M Pan, Z H Zhou. Image compression and encryption scheme based on 2D compressive sensing and fractional Mellin transform. Opt Commun. 2015, 343: $10-21$.

[37] X J Tong, M Zhang, Z Wang, J Ma. A joint color image encryption and compression scheme based on hyperchaotic system. Nonlinear Dyn. 2016, 84: 2333-2356.

[38] H Liu, D Xiao, R Zhang, Y S Zhang, S Bai. Robust and hierarchical watermarking of encrypted images based on compressive sensing. Signal Process: Image Commun. 2016, 45:41-51. [39] N R Zhou, J P Yang, C F Tan, S M Pan, Z H Zhou. Double-image encryption scheme combining DWT-based compressive sensing with discrete fractional random transform. Opt Commun. 2015, 354: 112-121.

[40] R Huang, K H Rhee, S Uchida. A parallel image encryption method based on compressive sensing. Multimed Tools Appl. 2014, 72(1): 71-93.

[41] Muhammad Ali Qureshi, M. Deriche. A new wavelet based efficient image compression 
algorithm using compressive sensing. Multimed Tools Appl. 2016, 75: 6737-6754.

[42] P Lu, Z Y Xu, X Lu, X Y Liu. Digital image information encryption based on compressive sensing and double random-phase encoding technique. Optik. 2013, 124: 2514-2518.

[43] D L Donoho. Compressed sensing. IEEE Trans. Inf. Theory 2006, 52: 1289-1306.

[44] E J Candes, J Romberg, T Tao. Robust uncertainty principles: exact signal reconstruction from highly incomplete frequency information. IEEE Trans. Inf. Theory 2006, 52: 489-509.

[45] L Yu, Jean Pierre Barbot, G Zheng, H Sun. Compressive sensing with chaotic sequence. IEEE Signal Process. Lett. 2010, 17: 731-734.

[46] Madalin Frunzete, L Yu, J Barbot, Adriana Vlad. Compressive sensing matrix designed by tent map, for secure data transmission. In: Proceedings of the IEEE Signal Processing: Algorithms, Architectures, Arrangements, and Applications (SPA), Poznan, September 2011, pp. 1-6

[47] Narendra K Pareek, Vinod Patidar, Krishan K Sud. Diffusion-substitution based gray image encryption scheme. Digit Signal Process. 2013, 23: 894-901.

[48] L Bao, Y C Zhou. Image encryption: generating visually meaningful encrypted images. Inf Sci. 2015, 324: 197-207. 